\title{
Real-Time Monitoring and Prediction of the Pilot Vehicle System (PVS) Closed-Loop Stability
}

\author{
Tanmay Kumar Mandal
}

Follow this and additional works at: https://researchrepository.wvu.edu/etd

\section{Recommended Citation}

Mandal, Tanmay Kumar, "Real-Time Monitoring and Prediction of the Pilot Vehicle System (PVS) ClosedLoop Stability" (2016). Graduate Theses, Dissertations, and Problem Reports. 6152.

https://researchrepository.wvu.edu/etd/6152

This Dissertation is protected by copyright and/or related rights. It has been brought to you by the The Research Repository @ WVU with permission from the rights-holder(s). You are free to use this Dissertation in any way that is permitted by the copyright and related rights legislation that applies to your use. For other uses you must obtain permission from the rights-holder(s) directly, unless additional rights are indicated by a Creative Commons license in the record and/ or on the work itself. This Dissertation has been accepted for inclusion in WVU Graduate Theses, Dissertations, and Problem Reports collection by an authorized administrator of The Research Repository @ WVU.

For more information, please contact researchrepository@mail.wvu.edu. 


\title{
Real-Time Monitoring and Prediction of the Pilot Vehicle System (PVS) Closed-Loop Stability
}

\section{Tanmay Kumar Mandal}

\author{
Dissertation submitted \\ to the Benjamin M. Statler College of Engineering and Mineral Resources \\ at West Virginia University \\ in partial fulfillment of the requirements for the degree of \\ Doctor of Philosophy in \\ Aerospace Engineering \\ Yu Gu, Ph.D., Chair \\ Marcello R. Napolitano, Ph.D. \\ Mario Perhinschi, Ph.D. \\ Xiaopeng Ning, Ph.D. \\ Sergiy Yakovenko, Ph.D.
}

Department of Mechanical and Aerospace Engineering

Morgantown, West Virginia

2016

Keywords: PIO, McRuer Model, UKF, Human Controlled System, Unmanned Aerial System

Copyright 2016 Tanmay Kumar Mandal 


\section{ABSTRACT \\ Real-Time Monitoring and Prediction of the Pilot Vehicle System (PVS) Closed-Loop Stability \\ Tanmay Kumar Mandal}

Understanding human control behavior is an important step for improving the safety of future aircraft. Considerable resources are invested during the design phase of an aircraft to ensure that the aircraft has desirable handling qualities. However, human pilots exhibit a wide range of control behaviors that are a function of external stimuli, aircraft dynamics, and human psychological properties (such as workload, stress, confidence, and sense of urgency). This variability is difficult to address comprehensively during the design phase and may lead to undesirable pilot-aircraft interaction, such as pilot-induced oscillations (PIO). This creates the need to keep track of human pilot performance in real-time to monitor the pilot vehicle system (PVS) stability.

This work focused on studying human control behavior using human-in-the-loop (HuIL) experiments to obtain information about the human controlled system (HCS) stability. The main focus of the dissertation is human control model parameter estimation To replicate different flight conditions, this study included time delay and actuator rate limiting phenomena, typical of actuator dynamics, during the experiments. To study human control behavior, this study employed the McRuer model for single-input singleoutput manual compensatory tasks. The McRuer model is a lead-lag controller with time delay which has been shown to adequately model manual compensatory tasks.

This dissertation presents a novel technique to estimate McRuer model parameters in real-time and associated validation using HuIL experiments to correctly predict HCS 
stability. The McRuer model parameters were estimated in real-time using an unscented Kalman filter (UKF) approach. The estimated parameters were then used to analyze the stability of the closed-loop HCS and verify them against the experimental data. 


\section{Declaration of Authorship}

I, Tanmay Kumar Mandal, declare that this thesis titled, 'Real-Time Monitoring and Prediction of the Pilot Vehicle System (PVS) Closed-Loop Stability' and the work presented in it are my own. I confirm that:

- This work was done wholly or mainly while in candidature for a research degree at this University.

- Where any part of this thesis has previously been submitted for a degree or any other qualification at this University or any other institution, this has been clearly stated.

- Where I have consulted the published work of others, this is always clearly attributed.

- Where I have quoted from the work of others, the source is always given. With the exception of such quotations, this thesis is entirely my own work.

- I have acknowledged all main sources of help.

- Where the thesis is based on work done by myself jointly with others, I have made clear exactly what was done by others and what I have contributed myself.

Signed:

Date: 
"Things are only impossible until they are not."

Star Trek 


\section{Acknowledgements}

This research was partially supported by NASA grant \# NNX12AM56A

I would like to thank my research advisor, $\mathrm{Dr}$. $\mathrm{Yu} \mathrm{Gu}$, for his guidance and support throughout this project. Dr. Gu, your insight and technical advice provided me with great opportunities to succeed and really learn to think about various research problems. I greatly appreciate your time and effort spent providing me with both technical and career advice.

I would like to thank my fellow researchers in Interactive Robotics Laboratory for assistance with the flight testing proram. The discussions, colaborative environment and constant encouragement to become a better group member and researcher, thank you.

I would also like to thank my committee members Dr. Marcello R. Napolitano, Dr. Mario Perhinschi, Dr. Xiaopeng Ning, and Dr. Sergiy Yakovenko. I appreciate your time and effort to review my thesis and provide helpful comments.

I would like to acknowledge the contribution of Stéphane D'Urso for helping me with $3 \mathrm{D}$ printing of numerous parts for my experiments.

Finally, I would like to thank my family who always taught me to be a better person. Thank you for your love and support. 


\section{Contents}

$\begin{array}{ll}\text { Abstract } & \text { ii }\end{array}$

Declaration of Authorship $\quad$ iv

Acknowledgements vi

Contents vii

List of Figures $\quad$ ix

List of Tables $\quad$ xi

Abbreviations $\quad$ xii

Symbols $\quad$ xiii

1 Introduction 1

1.1 Motivation and Literature Review . . . . . . . . . . . . . . . . 1

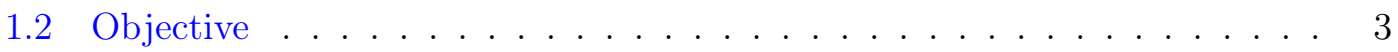

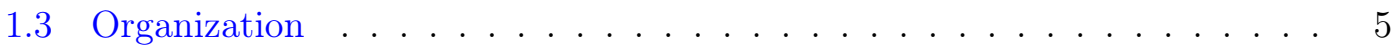

2 Online McRuer Model Parameter Estimation Using UKF 6

2.1 Introduction . . . . . . . . . . . . . . . . . . 6

2.2 Problem Formulation . . . . . . . . . . . . . . . . . . . . 7

2.2.1 Unscented Transformation and UKF . . . . . . . . . . . . . . . . 9

2.3 Simulations . . . . . . . . . . . . . . . . . . . . 11

2.4 Conclusions . . . . . . . . . . . . . . . . . . . . . 15

3 Human In-The Loop Simulation Studies $\quad 16$

3.1 Introduction . . . . . . . . . . . . . . . . . . . 16

3.2 Description of HuIL Experiment . . . . . . . . . . . . . . . . . . . . . . . . . . 17

3.2 .1 Experimental Setup . . . . . . . . . . . . . . . . 17

3.2 .2 Study Procedure . . . . . . . . . . . . . . . . . . . 22

3.3 McRuer Model Parameter Estimation . . . . . . . . . . . . . . . . 25

3.4 Estimation Results for HuIL Experiments with Time Delay . . . . . . . . 32 
3.5 Estimation Results for HuIL Experiments with Rate Limiting . . . . . . . 41

3.6 Prediction vs. Detection . . . . . . . . . . . . . . . . . 50

3.7 Statistical Anaysis . . . . . . . . . . . . . . . . . . . 50



4 Conclusions $\quad 53$

4.1 Limitations and Future Work . . . . . . . . . . . . . . . . . . . 54

A IRB Procedure and Approval 


\section{List of Figures}

2.1 Simulation setup. . . . . . . . . . . . . . . . . . . . . . 12

2.2 Time varying parameters values. . . . . . . . . . . . . . . . . . . 12

2.3 Human control command and pitch error value. . . . . . . . . . . . . . . 13

2.4 Pilot model parameter estimation results. . . . . . . . . . . . . . . . 13

2.5 Bode plot of true and estimated HCS at different time. . . . . . . . . . . . 14

3.1 HuIL experiment setup. . . . . . . . . . . . . . . . . . . 17

3.2 Bode plot of the pitch to joystick deflection transfer function (Eqn. 3.1) used for HuIL experiments. . . . . . . . . . . . . . . . . . . . . . . . . . . . . . . . 18

3.3 Error display as seen by subjects. . . . . . . . . . . . . . . . . . . . 19

3.4 Typical sum of sines signal used as desired pitch angle. . . . . . . . . . . . 21

3.5 Pitch error and joystick input for subject 1. . . . . . . . . . . . . . . . . 21

3.6 Pitch error and joystick input for subject 2. . . . . . . . . . . . . . . 22

3.7 Change in the standard deviation of the pitch error with successive trials. 23

3.8 Delayed (200 ms delay) joystick command HuIL experiment. . . . . . . . . . 24

3.9 Rate limited $(20 \mathrm{deg} / \mathrm{sec})$ joystick command. . . . . . . . . . . . . . . 24

3.10 Typical set of data used for estimation under nominal condition (no delay injection or rate limiting of joystick commands). . . . . . . . . . . . 26

3.11 Typical set of data used for estimation under nominal condition (no delay injection of rate limiting of joystick commands) . . . . . . . . . . . 26

3.12 Bode plot of the HCS at 35s after the start of the experiment. . . . . . . . 27

3.13 Bode plot of the HCS at 65s after the start of the experiment. . . . . . . . 27

3.14 Bode plot of the HCS at 90s after the start of the experiment. . . . . . . 28

3.15 Change in gain margin and phase cross over frequency with time. . . . . . 28

3.16 Change in phase margin and gain cross over frequency with time. . . . . . 28

3.17 Stability metric value of the closed-loop HCS with time. . . . . . . . . . 30

3.18 Comparison of estimated parameters for 5 different subjects for a single trial under nominal condition. . . . . . . . . . . . . . . . . . . . 31

3.19 Variation in gain margin and phase crossover frequency for same 5 different subjects. . . . . . . . . . . . . . . . . . 31

3.20 Variation in gain margin and phase crossover frequency for same 5 different subjects. . . . . . . . . . . . . . . . . . 31

3.21 Pitch error, joystick command and pitch rate for the HuIL experiment with $200 \mathrm{~ms}$ time delay injected. . . . . . . . . . . . . . . . 33

3.22 Estimation results for data shown in Figure 3.21. . . . . . . . . . . . . . 33

3.23 Change in gain margin and phase crossover frequency with time for experiment represented in Figure 3.21. . . . . . . . . . . . . . . . 34 
3.24 Change in phase margin and gain crossover frequency with time for experiment represented in Figure 3.21. . . . . . . . . . . . . . . 34

3.25 Stability metric value of closed-loop HCS with time for HuIL experiment with $300 \mathrm{~ms}$ time delay injection (Figure 3.21) . . . . . . . . . . 35

3.26 Recent minima and maxima selection for ROVER. Cyan vertical line represents time when delay was injected and black vertical line represents the current time till which ROVER analysis has been carried out. . . . . . . . 36

3.27 RIV for the HMS with time for HuIL experiment with 200ms time delay injection (Figure 3.21) . . . . . . . . . . . . . . . . 37

3.28 COF for HMS with time for HuIL experiment with 200ms time delay injection (Figure 3.21) . . . . . . . . . . . . . . . . . . . 38

3.29 Pitch, joystick command, and pitch rate data for HuIL experiment with $10 \mathrm{deg} / \mathrm{s}$ rate limiting. . . . . . . . . . . . . . . . . . . . . . . . . . . . . . 41

3.30 Estimation results for data shown in Figure 3.29 . . . . . . . . . . . . . 42

3.31 Change in gain margin and phase crossover frequency with time for the experiment represented in Figure 3.29 before the activation of rate limiting. 43

3.32 Change in phase margin and gain crossover frequency with time for experiment represented in Figure 3.29 before activation of rate limiting. . . . 43

3.33 Stability value of the closed-loop HCS with time for HuIL experiment before the activation of rate limiting. . . . . . . . . . . . . . . . . 4 43

3.34 Rate limit describing function characteristics. . . . . . . . . . . . . . . 44

3.35 Closed loop HCS with rate limiting. . . . . . . . . . . . . . . . . . 44

3.36 Nichols plot of the open loop system and inverse describing function at different time intervals. . . . . . . . . . . . . . . . . 45

3.37 Amplitude spectrum of pitch rate and forcing function for time interval $25-40 \mathrm{~s}$ for the data in Figure $3.29 \ldots \ldots$

3.38 Stability value of closed-loop HCS with time (after the activation of rate limiting) for HuIL experiment with $10 \mathrm{deg} / \mathrm{s}$ rate limiting. . . . . . . . . . 47

3.39 RIV for HCS with time for HuIL experiment after activation of $10 \mathrm{deg} / \mathrm{s}$ rate limit (Figure 3.29). . . . . . . . . . . . . . . . . . . . . . 48

3.40 COF for HCS with time for HuIL experiment after activation of $10 \mathrm{deg} / \mathrm{s}$ rate limit (Figure 3.29). . . . . . . . . . . . . . . . . . . . . . 48 


\section{List of Tables}

2.1 Mean and standard deviation of residual for each estimated parameters. $K_{p}, T_{\text {Lead }}, T_{\text {Lag }}$ are in arb. units. . . . . . . . . . . . 14

2.2 Stability margin comparison between true HCS and estimated HCS. . . . 14

3.1 Properties of desired pitch angle (sum of sines) . . . . . . . . . . . . 20

3.2 Definition of stability metric prediction regions. . . . . . . . . . . . . 29

3.3 ROVER thresholds. . . . . . . . . . . . . . . . . . . . . . 37

3.4 Comparison of the RPStab results with ROVER and DFT detection results for the HuIL experiment data with time delay presented in this section. 40

3.5 Comparison of total accuracy and HMO prediction/detection accuracy. . . 40

3.6 Limit cycle frequency at different time from Figure 3.36 . . . . . . . . . . 46

3.7 Comparison of the RPLC method results with ROVER and DFT detection results for HuIL experiment with rate limiting presented in this section. . 49

3.8 Comparison of total accuracy and HMO prediction/detection accuracy for HuIL experiments with rate limiting . . . . . . . . . . . . . . . . . 49 


\section{Abbreviations}

$\begin{array}{ll}\text { COF } & \text { Combined Oscillation Flag } \\ \text { EKF } & \text { Extended Kalman Filter } \\ \text { FP } & \text { False Positive } \\ \text { FN } & \text { False Negative } \\ \text { HCS } & \text { Human Controlled System } \\ \text { HuIL } & \text { Human In the Loop } \\ \text { KF } & \text { Kalman Filter } \\ \text { LHP } & \text { Left Hand Plane } \\ \text { LOC } & \text { Loss Of Control } \\ \text { OLOP } & \text { Open Loop Onset Point } \\ \text { PIO } & \text { Pilot Induced Oscillation } \\ \text { PVS } & \text { Pilot Vehicle System } \\ \text { RHP } & \text { Right Hand Plane } \\ \text { RIV } & \text { Rover Integer Value } \\ \text { ROVER } & \text { Real-Time Oscillation Verifier } \\ \text { RPLC } & \text { Real-Time Prediction of Limit Cycle } \\ \text { RPStab } & \text { Real-Time Prediction of Stability } \\ \text { TN } & \text { True Negative } \\ \text { TP } & \text { True Positive } \\ \text { UKF } & \text { Unscented Kalman Filter } \\ \text { wDFT } & \text { Windowed Discrete Fourier Technique } \\ & \end{array}$




\section{Symbols}

$\delta_{e}(s) \quad$ elevator deflection in frequency domain

$\delta_{j}(s) \quad$ joystick deflection in frequency domain

$\delta_{p}(s) \quad$ elevator deflection command in frequency domain

st $\quad$ sampling time

$\omega_{B W} \quad$ frequency bandwidth

$\delta_{s}(s) \quad$ elevator stick deflection in frequency domain

$\theta(s) \quad$ aircraft pitch angle in frequency domain

$\tau_{p} \quad$ phase delay parameter

$\tau_{i} \quad$ time delay injected in HuIL experiments

$\phi(\omega) \quad$ phase angle at frequency $\omega$ in Bode plot

$\omega_{180}$ frequency at $180^{\circ}$ phase angle

$\omega_{B W_{\gamma}} \quad$ flight path bandwidth

$\omega_{L} C \quad$ limit cycle frequency

$q(s) \quad$ aircraft pitch rate in frequency domain

$E(S)$ pitch error in frequency domain

$G M_{k} \quad$ gain margin at time step $k$

$K_{p} \quad$ pilot gain

$P(S) \quad$ pilot output in frequency domain

$P M_{k} \quad$ phase margin at time step $k$

$S_{k} \quad$ stability value at time step $k$

$T_{L} \quad$ pilot lead

$T_{1} \quad$ pilot lag, Neal-Smith criterion

$\tau \quad$ pilot intrinsic delay

$\omega_{c r} \quad$ aircraft-pilot crossover frequency

$P R_{180}$ phase rate at $180^{\circ}$ phase angle 
$\begin{array}{ll}\omega_{\text {onset }} & \text { onset frequency } \\ R_{L} & \text { maximum rate permitted }\end{array}$ 


\section{Chapter 1}

\section{Introduction}

\subsection{Motivation and Literature Review}

$\mathrm{B}$ efore the Wright brothers, aerospace engineers already knew that an airfoil shaped cross-section of a wing would provide sufficient lift to fly heavier-than-air machines. However, the Wright brothers' breakthrough invention of three-axis aircraft control made it possible for a human pilot to fly and steer an aircraft safely [1]. Even today, pilot-vehicle interaction receives much attention during the design phase of an aircraft. However, it is difficult to foresee all the possible ways a human pilot can interact with an aircraft due to a human pilot's high degree of variability, non-linearity, and adaptability. The complex nature of a human operator demands a collective approach of analysis from the standpoint of control theory, psychology, neurology, and biology of human sensors, such as the eye and vestibular system $[2,3,4]$. If not properly designed to mitigate various handling issues regarding pilot-vehicle interaction, loss of control (LOC) events such as pilot induced oscillations (PIOs) [5] could happen in which the pilot loses control of the aircraft. There have been several high profile PIO incidents [6, 7] in aviation history, which emphasizes the need to better understand human pilot control characteristics as a part of the overall pilot vehicle system (PVS) to reduce the occurrence of such incidents. The Military Standard for Flying Qualities of Piloted Aircraft (MIL-STD 1797A) [8] defines PIO as "sustained or uncontrollable oscillations resulting from efforts 
of the pilot to control the aircraft." It has been observed that recent advances in digital fly-by-wire technology with augmented controls have led to an increase in PIO events [9, 10, 11, 12, 13]. The increasing complexity of digital fly-by-wire technology has made it increasingly difficult to fully understand the stability properties of the PVS under various circumstances.

A mathematical model that can capture the control characteristics of a human pilot can provide important information regarding the stability margin of the PVS and help predict the onset of instabilities. The first systematic attempt to study the manual control of dynamic systems started in the 1940s. This earlier work began by studying humans as a controller of single-variable, single-display linear time-invariant systems. However, this was insufficient to generalize for most complex dynamic systems, such as aircraft or cars [14, 15]. For a particular flying condition consisting of a simple task, a human pilot can be satisfactorily represented as a quasi-linear system with a linear component consisting of a corresponding gain, lead, lag, time delay, and nonlinear remnant [16]. This quasi-linear model may not necessarily replicate the human control output exactly, but is capable of giving basic information about the behavioral properties of the human controller for control tasks in systems such as aircraft or cars [17]. Such behavioral properties include the system order, bounds on natural frequency, and margins $[18,3,4,19]$. The most widely used quasi-linear models for human operators resulted from the work of Russell, et al. [17], based on extensive experimental studies on different type of systems. The quasi-linear pilot model consists of parameters that represent the inherent limitations of human control, such as control bandwidth and delay in the human neuromuscular system. For most single-input single-output problems, this quasi-linear pilot model, which is essentially a lead-lag compensator with delay, has been successfully used for human control analysis [20,21, 22]. Another way of modeling human control is to represent it by optimal controller [23, 24], this requires that the human controller is highly skilled and operating conditions are invariant. However, during flight, many factors change, such as wind gust, aircraft dynamics (for example change in fuel weight or control modes), and the state of human operator. All these factors may lead to difficulties in finding an optimal control strategy. This makes McRuer model better suited for the study of general human control behavior[17]. 


\subsection{Objective}

The objective of this research was to study, quantify, and predict the stability characteristics in real-time of single-input single-output manual compensatory tasks. Such real-time estimation and analysis techniques could in the future be used to notify a pilot of the onset of certain LOC events. However, factors such as high variability in the control behavior among human operators, as well as their non-linearity and adaptability, can make it challenging to develop any type of real-time human controller performance monitoring or prediction framework. For this research, the human controller was modeled with a McRuer $[2,3,4]$ model, with simulated pitch error as the input and joystick deflection as the output. Only reactive compensatory control was considered in this research - that is, the human controller aimed to reduce pitch angle error. If the task involves only pitch error reduction and the only feedback available to the human controller is the pitch error information, then the human controller behavior is predominantly reactive and compensatory[2]. The other major type of control is predictive [2], which was not studied during this effort. In predictive control human controller formulates a model of the evolving input to generate control commands instead of relying solely on the error feedback.

Real-time stability assessment of the closed-loop HCS requires real-time estimation of the McRuer model parameters. Current McRuer model estimation techniques place great emphasis on batch estimation techniques (such as least squares and maximum likelihood) [22][25][26], in which researchers examine the flight data from a particular flight phase and calculate the McRuer model parameters in an average sense post-flight; i.e., estimation is carried out after the flight data is obtained and not in real-time. The pilot model obtained using batch estimation is then used to quantify the stability margins of the closed-loop HCS [22]. Although this method provides valuable insights to human pilot characteristics, it neglects the variable nature of human behavior during the task which can be represented by the change in McRuer model parameters. This dissertation presents an estimation technique based on an unscented Kalman filtering (UKF) method that estimates the McRuer model parameters in real-time. UKF is an extension of 
Kalman filter (KF) technique to non-linear state estimation and can be implemented in real-time using predictor-corrector hierarchy [27]. The advantages of this technique are immediately apparent in terms of being able to capture the variable nature of human control. It also provides a real-time tool to monitor and predict the stability of the closed-loop HCS. Other real-time techniques include the windowed maximum likelihood estimator [28] and wavelet technique [29], which are susceptible to the length of window of observation (i.e., the segment of data used for analysis and can induce delay in estimation) and measurement noise used for feedback to improve the estimation. In contrast, the UKF is easy to implement, is iterative, does not require a highly accurate representation of initial conditions and is relatively resistant to measurement noises[27]. This property makes UKF an ideal tool for real-time estimation of McRuer model parameters.

Previously extended Kalman filter (EKF) [30] had been used for real-time estimation of McRuer model parameters but it failed to achieve satisfactory results due to nonlinearity of the estimation problem and lack of validation of the estimated parameters. Johnson et. al. concluded that the pilot model parameters can be estimated with small variance (high confidence) in real-time if the magnitude of the process noise is sufficiently small. However, if the low process noise assumption fails and the assumed initial conditions are drastically different to the real values (which are unknown), the EKF diverged rapidly. In addition, EKF uses first order linearization for non-linear estimation where as UKF uses a statistical linearization approach based on unscented transformation and is of second order. This makes UKF more accurate and easier to implement as compared to EKF. This dissertation presents a robust real-time estimator formulation based on UKF.

In summary, the major contributions of this dissertation are:

1. Estimation of human control model parameters using UKF in real-time

2. Prediction of instability in a closed-loop human-machine system for pitch control using HuIL experiments. 


\subsection{Organization}

The rest of this dissertation is organized as follows. Chapter 2 provides details of the UKF formulation for the real-time McRuer model parameter estimation and corresponding simulation results. Chapter 3 presents the HuIL experiments undertaken to validate the estimator with actual human control data. Finally, Chapter 4 provides the conclusion for this dissertation. 


\section{Chapter 2}

\section{Online McRuer Model Parameter Estimation Using UKF}

\subsection{Introduction}

$\mathrm{I}$

n this chapter, a UKF based [31] real-time McRuer model parameter identification algorithm is presented for a single-input single-output manual control tasks. The UKF estimates the parameters of the McRuer model using human control inputs and simulated pitch error data. The UKF algorithm was tested in simulations and was shown to estimate the parameters with sufficient accuracy.

The rest of the chapter is organized as follows. Section 2.2 presents the formulation of equations which were used in UKF for McRuer model parameter estimation. Section 2.3 presents simulation result and finally, Section 2.4 contains discussion about the observations obtained and presents the conclusion. 


\subsection{Problem Formulation}

This section provides a detailed description of the formulation used for estimation purposes. The McRuer model [32] is given by:

$$
P(s)=K_{\text {pilot }} \frac{T_{\text {Lead }} s+1}{T_{\text {Lag }} s+1} \mathrm{e}^{-\tau s} E(s)
$$

Where $\mathrm{P}(\mathrm{s})$ is the frequency domain human control command, $\mathrm{E}(\mathrm{s})$ is the frequency domain error being minimized by the human controller, $K_{\text {pilot }}$ is the McRuer model gain, $T_{\text {Lead }}$ is the lead factor, $T_{L a g}$ is the lag factor, and $\tau$ is the delay intrinsic to human controller. The parameters that need to be estimated are $K_{\text {pilot }}, T_{\text {Lead }}, T_{\text {Lag }}$, and $\tau$. Eqn. 2.1 can be written in time domain as

$$
\begin{aligned}
\dot{X}_{\text {pilot }}(t) & =\frac{1}{T_{\text {Lag }}} X_{\text {pilot }}(t)+e(t-\tau) \\
p(t) & =\frac{K_{\text {pilot }}\left(T_{\text {Lag }}-T_{\text {Lead }}\right)}{T_{\text {Lag }}^{2}} X_{\text {pilot }}(t)+\frac{K_{\text {pilot }} T_{\text {Lead }}}{T_{\text {Lag }}} e(t-\tau)
\end{aligned}
$$

In Eqn. $2.2 p(t)$ is time domain human control output, which can be measured and $e(t-\tau)$ is the time domain error delayed by $\tau$ seconds. $X_{\text {pilot }}(t)$ is a $1 \times 1$ human control state, which cannot be measured directly and needs to be solved to use the differential equation in Eqn. 2.2 to calculate the control output $p(t)$. The time delay is approximated by a first order Pade approximation (Eqn. 2.3) to make the McRuer model amenable to linear system analysis methods (such as bode analysis and state space expression). The new transfer function is given by Eqn. 2.4:

$$
\begin{gathered}
\mathrm{e}^{-\tau s}=\frac{2-\tau s}{2+\tau s} \\
P(s)=\frac{-\tau T_{\text {Lead }} K_{\text {pilot }} s^{2}+\left(2 T_{\text {Lead }}-\tau\right) K_{\text {pilot }} s+2 K_{\text {pilot }}}{\tau T_{\text {Lag }} s^{2}+\left(2 T_{\text {Lag }}+\tau\right) s+2}
\end{gathered}
$$


Converting Eqn. 2.4 to a controllable canonical state space form gives Eqn. 2.5

$$
\begin{aligned}
\dot{X}_{\text {pilot }}(t) & =A_{\text {pilot }} X_{\text {pilot }}(t)+B_{\text {pilot }} e(t) \\
p(t) & =C_{\text {pilot }} X_{\text {pilot }}(t)+D_{\text {pilot }} e(t) \\
A_{\text {pilot }} & =\left[\begin{array}{cc}
\frac{-\left(2 T_{\text {Lag }}+\tau\right)}{\tau T_{\text {Lag }}} & \frac{-2}{\tau T_{\text {Lag }}} \\
1 & 0
\end{array}\right], B_{\text {pilot }}=\left[\begin{array}{l}
1 \\
0
\end{array}\right] \\
C_{\text {pilot }} & =\left[\begin{array}{ll}
\beta_{1} & \beta_{2}
\end{array}\right], D_{\text {pilot }}=b_{0}
\end{aligned}
$$

Where $X_{\text {pilot }}(t)$ is the $2 \times 1$ human control state vector (it changed dimension due to Pade approximation), $A_{\text {pilot }}$ is the $2 \times 2$ system matrix, $B_{\text {pilot }}$ is the $2 \times 1$ control matrix, $C_{\text {pilot }}$ is the $1 \times 2$ output matrix, and $D_{\text {pilot }}$ is the $1 \times 1$ feed-forward matrix. The parameters $b_{0}, b_{1}, b_{2}, \beta_{1}, \beta_{2}, a_{1}$, and $a_{2}$ are defined in the following equation.

$$
\begin{aligned}
& b_{0}=\frac{-T_{\text {Lead }} K_{\text {pilot }}}{T_{\text {Lag }}}, b_{1}=\frac{\left(2 T_{\text {Lead }}-\tau\right) K_{\text {pilot }}}{\tau T_{\text {Lag }}}, b_{2}=\frac{2 K_{\text {pilot }}}{\tau T_{\text {Lag }}} \\
& \beta_{1}=b_{1}-D_{\text {pilot }} a_{1}, \beta_{2}=b_{2}-D_{\text {pilot }} * a_{2} \\
& a_{1}=\frac{\left(2 T_{\text {Lag }}+\tau\right)}{\tau T_{\text {Lag }}}, a_{2}=\frac{2}{\tau T_{\text {Lag }}}
\end{aligned}
$$

Discretizing Eqn. 2.5 gives the following:

$$
\begin{aligned}
X_{\text {pilot }_{k}} & =A_{\text {pilot }_{d}} X_{\text {pilot }_{k-1}}+B_{\text {pilot }_{d}} e_{k-1} \\
A_{\text {pilot }_{d}} & =\mathrm{e}^{A_{\text {pilot } T_{s}}, B_{\text {pilot }_{d}}=A_{\text {pilot }}^{-1}\left(A_{\text {pilot }_{d}}-I\right) B_{\text {pilot }}, T_{s}=\text { Sampling time }} \\
p_{k} & =C_{\text {pilot }} X_{\text {pilot }_{k}}+D_{\text {pilot }} e_{k-1}
\end{aligned}
$$

In Eqn. $2.7 A_{\text {pilot }_{d}}$ is the $2 \times 2$ discretized system matrix and $B_{\text {pilot }_{d}}$ is the $2 \times 1$ discretized control matrix. For simulation a $T_{s}$ value of $0.01 \mathrm{~s}$ was used and $k$ was the time step at time, $k T_{s}$. Similarly $k-1$ was the time step at $(k-1) T_{s}$. It is evident from Eqn. 2.5 that the equation was non-linear in the parameters to be estimated due to presence of terms like $\tau T$. For simulation studies, the following pitch system given by 
Eqn. 2.8 was used:

$$
\begin{aligned}
& X_{\text {pitch }_{k}}=A_{\text {pitch }_{d}} X_{\text {pitch }_{k-1}}+B_{\text {pitch }_{d}} p_{k-1} \\
& \theta_{k}=C_{p i t c h} X_{p i t c h k-1} \\
& A_{\text {pitch }}=\mathrm{e}^{A_{\text {pitch }} T_{s}}, B_{\text {pitch }}=A_{\text {pitch }}^{-1}\left(A_{\text {pitch }}-I\right) B_{\text {pitch }} \\
& A_{\text {pitch }}=\left[\begin{array}{ccc}
-3.59 & -22.250 & \\
1 & 0 & 0 \\
0 & 1 & 0
\end{array}\right], B_{\text {pitch }}=\left[\begin{array}{l}
1 \\
0 \\
0
\end{array}\right] \\
& C_{\text {pitch }}=\left[\begin{array}{lll}
0 & -15.44 & -59.93
\end{array}\right], D=0
\end{aligned}
$$

In Eqn. $2.8 X_{\text {pitch }_{k}}$ is the state of the pitch plant at time step $k, A_{\text {pitch }}$ is the $3 \times 3$ discretized pitch plant matrix, $B_{\text {pitch }}$ is the $3 \times 1$ discretized plant control matrix, $C_{\text {pitch }}$ is the $3 \times 1$ output matrix, $D_{\text {pitch }}$ is the feed-forward gain, $\theta_{k}$ is the pitch output at time step $k$.

The equations presented in this section were used in the unscented transform central to UKF presented in next section.

\subsubsection{Unscented Transformation and UKF}

The unscented transformation is used to handle non-linearity in $Y=f(X)$, where $X$ and $Y$ are $L \times 1$ vectors (here, $L$ is the length of the state vector), and $f$ is a $L \times 1$

vector-valued function. Here, $X$ is a random variable with mean, $\bar{X}$, and covariance, $P_{x}$. Unscented transformation is central to UKF because it provides a statistical alternative to analytical linearization used in EKF.

A great deal of work has been done on UKF for nonlinear estimation problems, and details can be found in the existing literature [33, 27, 34]. For the McRuer model estimation problem, it is assumed that the process and measurement noise terms were additive, as in:

$$
\begin{aligned}
X_{k} & =f\left(X_{k-1}, e_{k-1}, p_{k-1}\right)+w_{k-1} \\
Y_{k} & =h\left(X_{k}, e_{k}, p_{k}\right)+v_{k}
\end{aligned}
$$


Where $X$ is the state to be estimated, which is defined as

$$
X=\left[a_{1}, a_{2}, b_{0}, b_{1}, b_{2}, X_{\text {pilot }}\right]^{T}
$$

In addition, $Y_{k}$ is the estimated human control command at time step, $f$ is the non-linear state propagation function, $h$ is the non-linear measurement function. Terms $a_{1}, a_{2}, b_{0}$, $b_{1}, b_{2}$ are defined in Eqn. 2.6. The process $(w)$ and measurement $(v)$ noise are considered uncorrelated, white, and Gaussian with zero mean and known covariance matrices, $Q$ and $R$, respectively, as in Eqn. 2.11:

$$
\begin{aligned}
& w_{k} \sim N\left(0, Q_{k}\right) \\
& v_{k} \sim N\left(0, R_{k}\right) \\
& E\left[w_{k} v_{k}^{T}\right]=0
\end{aligned}
$$

In the above equation $N$ represents a normal distribution and $E$ is the expected operator.

The UKF process and measurement equation are then given by the following:

$$
\begin{gathered}
{\left[\begin{array}{c}
a_{1_{k}} \\
a_{2_{k}} \\
b_{0_{k}} \\
b_{1_{k}} \\
b_{2_{k}} \\
X_{\text {pilot }_{k}}
\end{array}\right]=\left[\begin{array}{c}
a_{1_{k-1}} \\
a_{2_{k-1}} \\
b_{0_{k-1}} \\
b_{1_{k-1}} \\
b_{2_{k-1}} \\
A_{\text {pilot }_{d}} X_{\text {pilot }_{k-1}}+B_{\text {pilot }_{d}} e_{k-1}
\end{array}\right]+w_{k-1}} \\
Y_{k}=\left[C_{\text {pilot }_{\text {pilot }}}+D_{\text {pilot }_{\text {pot }} e_{k}}\right]+v_{k}
\end{gathered}
$$

At each time step $k$, a set of sigma-points are generated from the state estimate and covariance at time step $k-1[27]$. At each time step, the state predicted using Eqn. 2.9 is corrected (or "updated") using the actual human control command measured at that time step. The extent of correction is dependent on the Kalman gain $\left(K_{k}\right)$ calculated 
from the UKF algorithm [27]. The update equation for $X$ is given by:

$$
\hat{X}_{k}=\hat{X}_{k \mid k-1}+K_{k}\left(Y_{k}-\hat{Y}_{k \mid k-1}\right)
$$

Where $Y_{k}=$ pilot $_{k}$ is the actual human control command measured at time step $k$. In Eqn. 2.13, $\hat{X}_{k}$ is the updated state at time step $k, \hat{X}_{k \mid k-1}$ is the predicted state at time step $k$ from Eqn. 2.9, and $\hat{Y}_{k \mid k-1}$ is the predicted output at time step $k$ from Eqn. 2.9. The parameters $K_{\text {pilot }}, T_{\text {Lead }}, T_{\text {Lead }}$, and $\tau$ can then be obtained from the state $X$ using Eqn. 2.5. The accuracy of the estimated parameters were then determined using simulations, details of which are presented in the following section.

\subsection{Simulations}

To validate the estimation algorithm, a simulation was setup in Simulink ${ }^{\circledR}$ to generate data. This section provides a description of the simulation setup and results. The simulation model consisted of a pitch transfer function and McRuer model implemented in state space form (provided in Eqn. 2.7) to close the loop. For all simulations, the values of $K_{\text {pilot }}, T_{\text {Lead }}$, and $T_{\text {Lag }}$ were varied gradually; however, the value of $\tau$ was kept constant. It was assumed that since the human controller was focused on the task performed the intrinsic delay would not vary significantly [32]. The simulations were performed for various values of $\tau$ and varying degree of changes in $K_{\text {pilot }}, T_{\text {Lead }}$, and $T_{\text {Lag }}$ parameters. Figure 2.1 shows the simulation scheme developed to generate the simulation data.

The input (i.e., the reference signal to the system) was a sum of sines that covered the frequency range of human control $(0.1-20 \mathrm{rad} / \mathrm{s})$ [28]. Figure 2.2 and Figure 2.3 shows one example of the time-varying parameters and measurement values used in a typical simulation. Figure 2.4 shows the estimation result obtained for the simulation data shown in Figure 2.3. 


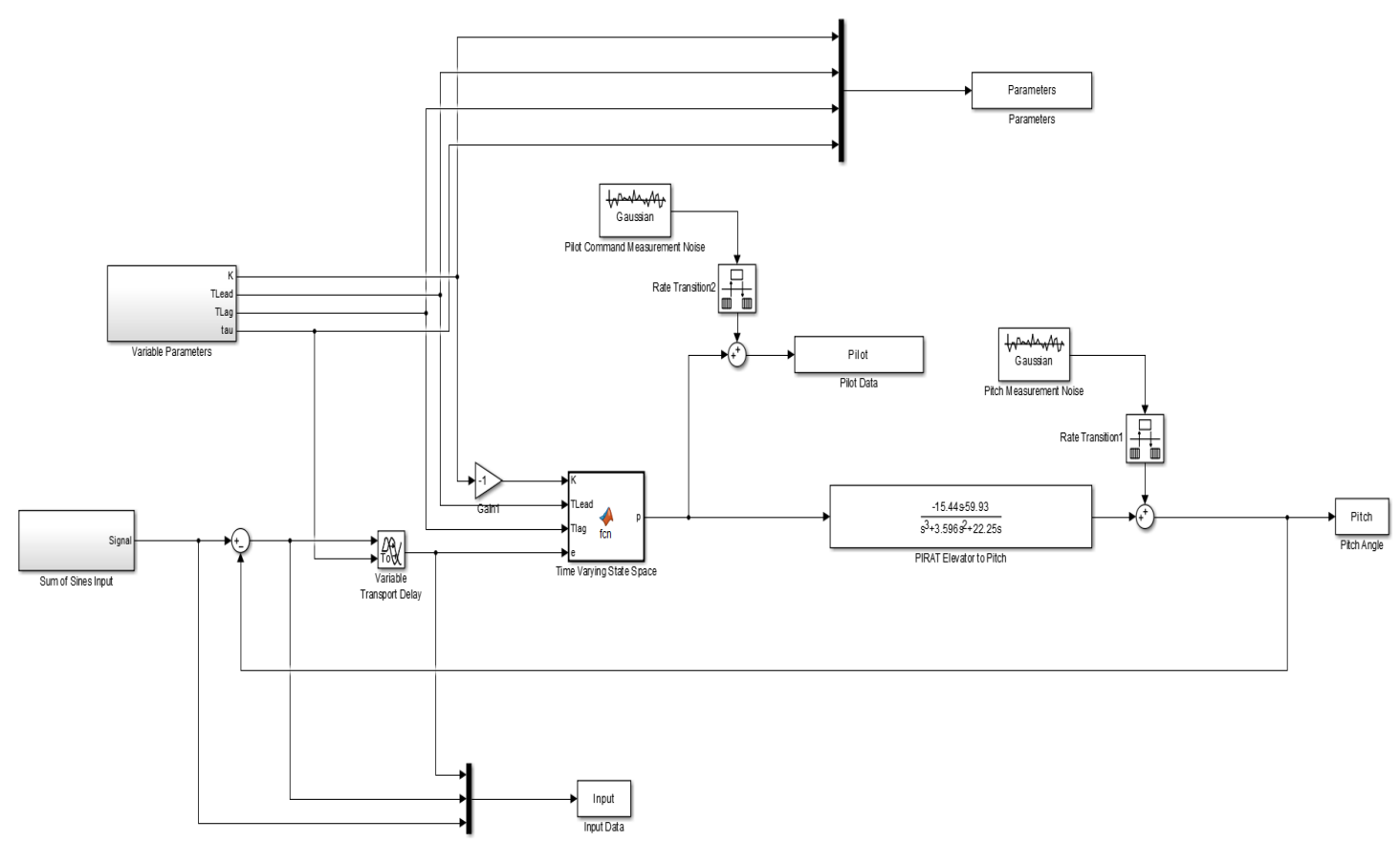

Figure 2.1: Simulation setup.

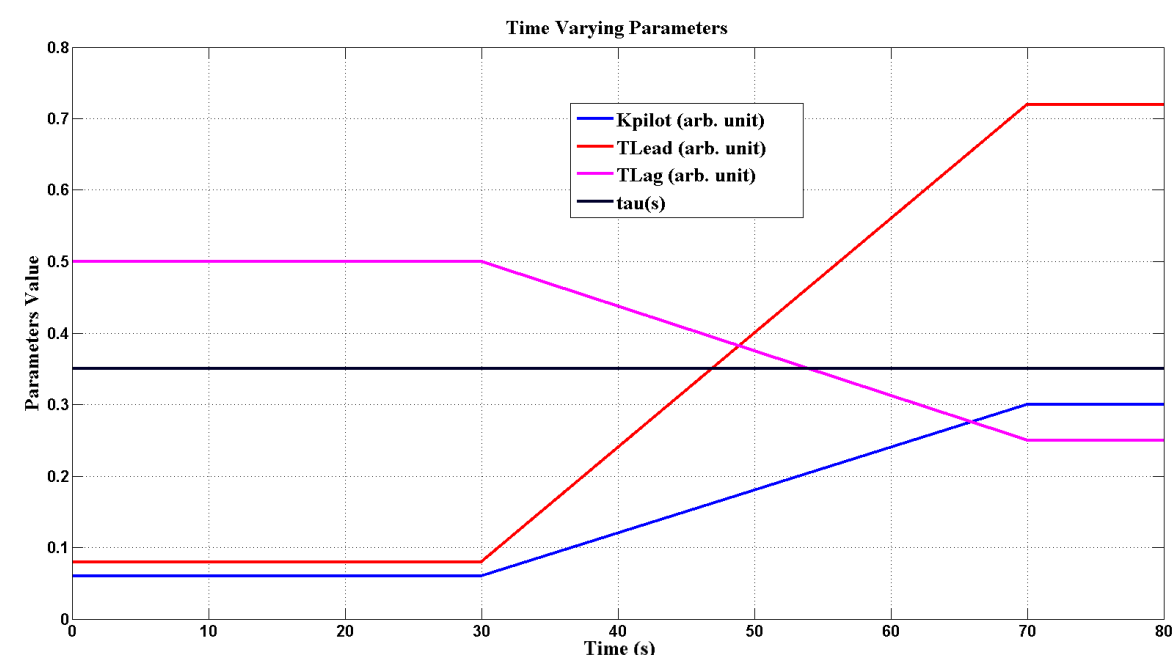

FIGURE 2.2: Time varying parameters values.

The results showed that the developed UKF formulation had good performance regarding tracking changes in the McRuer model parameters under this particular simulation setup. The mean and standard deviation of the residual for each parameter are provided in Table 2.1. However, it was necessary to quantify the performance of the estimator under modeling error because human controllers exhibit highly variable behavior and may not always behave as the McRuer lead-lag model. To study the effect of modeling errors, further simulations were performed with a human control model different to the McRuer model, while using the UKF to estimate parameters for a McRuer model. 


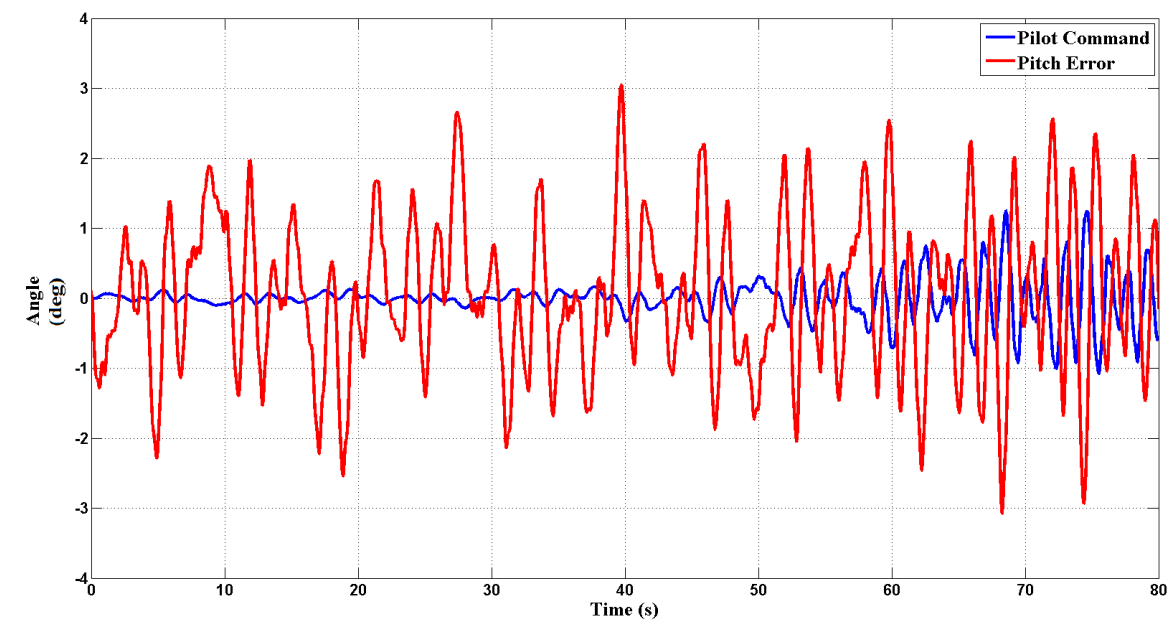

Figure 2.3: Human control command and pitch error value.
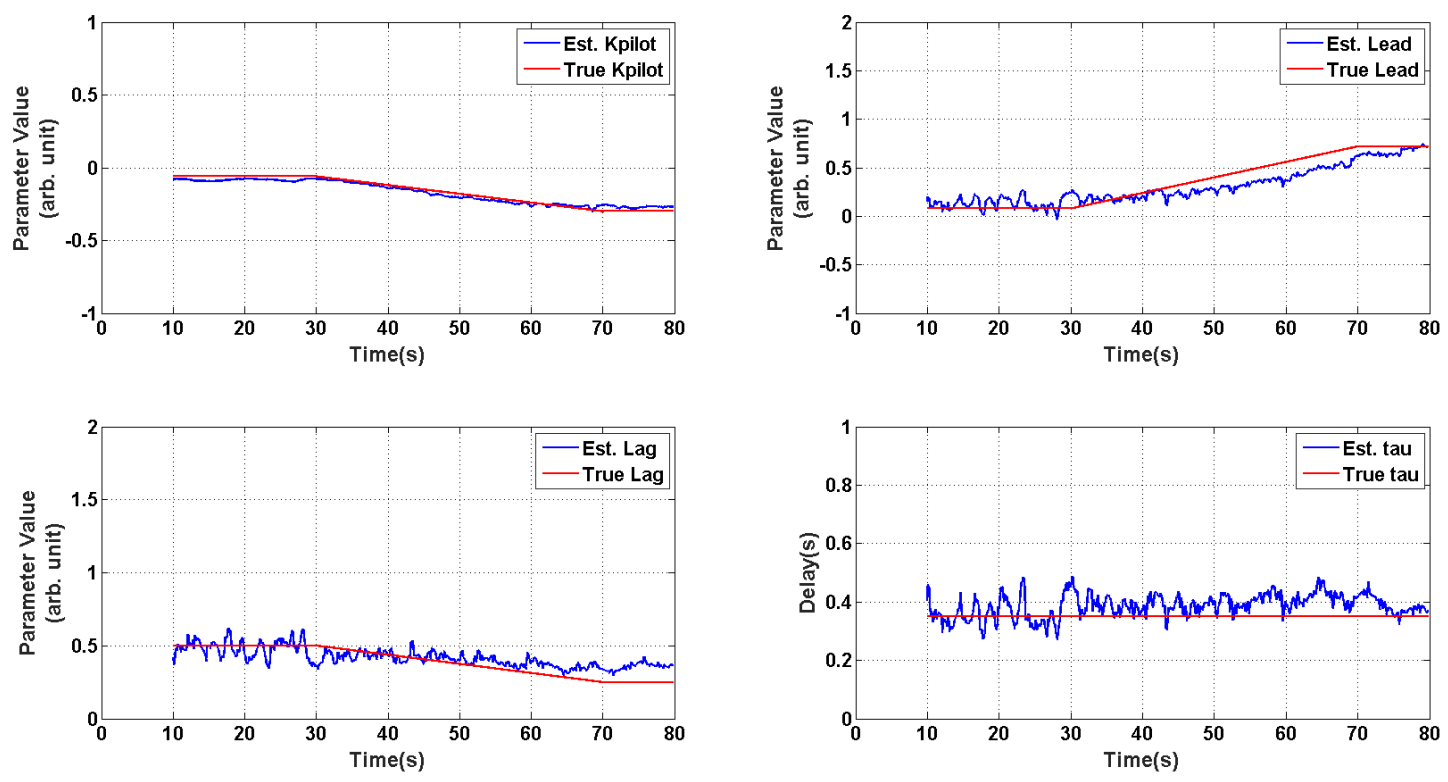

FIGURE 2.4: Pilot model parameter estimation results.

The "true model" used to generate the simulation data is given by:

$$
P(s)=\frac{K_{\text {pilot }}}{T_{\text {Lag }} s+1} \mathrm{e}^{-\tau s}
$$

In Eqn. 2.14, the parameters $K_{\text {pilot }}, T_{\text {Lag }}$, and $\tau$ were varied according to Figure 2.2; however, parameter $T_{\text {Lead }}$ was not used. To quantify the performance of the UKF estimator, the gain margin and phase margin of the HCS obtained using the estimated McRuer model were compared with that of the HCS obtained using the" true model". Figure 2.5 shows the Bode plot for the true HCS and estimated HCS at 25, 60, and 80 s. 
TABLE 2.1: Mean and standard deviation of residual for each estimated parameters. $K_{p}, T_{\text {Lead }}, T_{\text {Lag }}$ are in arb. units.

\begin{tabular}{ccc}
\hline Parameters & Mean & Std. \\
\hline$K_{p}$ & 0.0170 & 0.0083 \\
$T_{\text {Lead }}$ & 0.0470 & 0.0110 \\
$T_{\text {Lag }}$ & 0.0330 & 0.0097 \\
$\tau$ & $0.0810 \mathrm{~s}$ & $0.0166 \mathrm{~s}$ \\
\hline
\end{tabular}
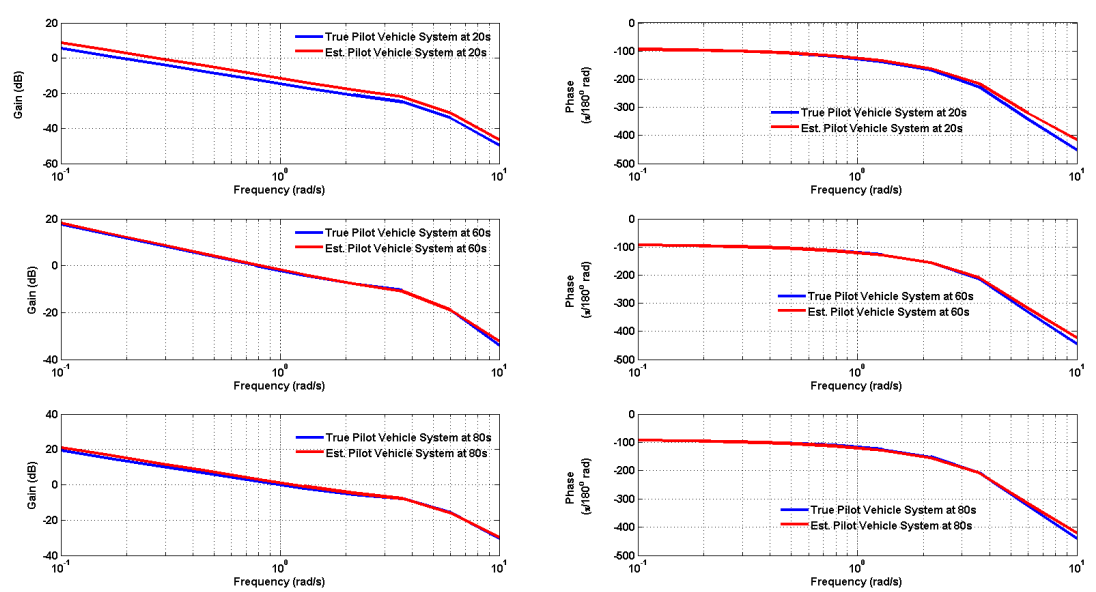

Figure 2.5: Bode plot of true and estimated HCS at different time.

TABLE 2.2: Stability margin comparison between true HCS and estimated HCS.

\begin{tabular}{ccc}
\hline & True HCS & Est. HCS \\
\hline At 20 s & & \\
Gain Margin & $22.1 \mathrm{~dB}$ at $2.46 \mathrm{rad} / \mathrm{s}$ & $19.8 \mathrm{~dB}$ at $2.66 \mathrm{rad} / \mathrm{s}$ \\
Phase Margin & $82.9^{\circ}$ at $0.188 \mathrm{rad} / \mathrm{s}$ & $80.3^{\circ}$ at $0.275 \mathrm{rad} / \mathrm{s}$ \\
At 60 s & & \\
Gain Margin & $9.25 \mathrm{~dB}$ at $2.79 \mathrm{rad} / \mathrm{s}$ & $9.48 \mathrm{~dB}$ at $2.85 \mathrm{rad} / \mathrm{s}$ \\
Phase Margin & $67.6^{\circ}$ at $0.769 \mathrm{rad} / \mathrm{s}$ & $65.5^{\circ}$ at $0.814 \mathrm{rad} / \mathrm{s}$ \\
At 80 s & & \\
Gain Margin & $6.97 \mathrm{~dB}$ at $2.94 \mathrm{rad} / \mathrm{s}$ & $6.58 \mathrm{~dB}$ at $2.87 \mathrm{rad} / \mathrm{s}$ \\
Phase Margin & $63.9^{\circ}$ at $0.984 \mathrm{rad} / \mathrm{s}$ & $55.6^{\circ}$ at $1.15 \mathrm{rad} / \mathrm{s}$ \\
\hline
\end{tabular}

It can be seen from Figure 2.5 that the Bode plot for the true HCS and estimated HCS at time steps before the change in parameters, during the change in parameters, and after the change in parameters matched very closely. Table 2.2 presents the gain and phase margin for true and estimated HCS. 
It is clear from Table 2.2 that the true HCS and estimated HCS had very similar stability characteristics . That is, they have similar phase and gain margins. Similar re-

sults were also obtained for simulations performed with $\frac{0.2 K_{\text {pilot }}}{s} \mathrm{e}^{-\tau s}$ and $\frac{2 K_{\text {pilot }}}{T_{\text {Lag }} s^{2}+s+1} \mathrm{e}^{-\tau s}$ as true human control models.

The ability to predict the stability characteristics of the true HCS is important to predicting unfavorable human-machine interactions. The simulation results showed that the UKF-based McRuer model parameter estimator provided good estimation of HCS stability characteristics, even under substantial modeling errors.

\subsection{Conclusions}

A UKF-based technique for estimating McRuer model parameters in real-time was presented. The estimator formulated in this chapter was shown to estimate human control parameters with good accuracy in simulation studies. It was also shown that under modeling errors, the estimator was still able to estimate parameters for the McRuer model such that the stability characteristics of the estimated HCS were similar to a true HCS model used during simulation.

The estimator developed in this chapter still needs to be validated with actual human data under a controlled environment. This can be achieved by undertaking HuIL experiments for various scenarios and comparing the prediction results with experimental data. The following chapter provides detail of the HuIL experiments used for this research. 


\section{Chapter 3}

\section{Human In-The Loop Simulation}

\section{Studies}

\subsection{Introduction}

$\mathrm{C}$

ontrolled experiments were needed to ensure that the estimator developed in Section 2.2 was performing as expected. HuIL experiment is one technique that allows the researcher to properly control the various factors affecting the human control behavior. HuIL experiment gives researchers better control over the type of system used, control tasks, and feedback to the human controller, while still enabling access to actual human control commands in a controlled manner. Therefore, HuIL experiment studies were used to assess the capabilities and effectiveness of the online parameter estimation technique developed in the previous chapter for a wide range of scenarios.

The objective of this chapter is to use the UKF-based algorithm developed in the previous chapter for estimating human control model parameters during under different conditions, such as time delay injection and actuator rate limiting, in order to establish its validity in characterizing the stability of a single-input single-output manual compensatory task. This chapter is divided into five major sections. Section 3.2 provides a description of the HuIL setup, experimental procedures, and data analysis techniques. Section 3.3 provides the estimation results obtained using experimental data from various 


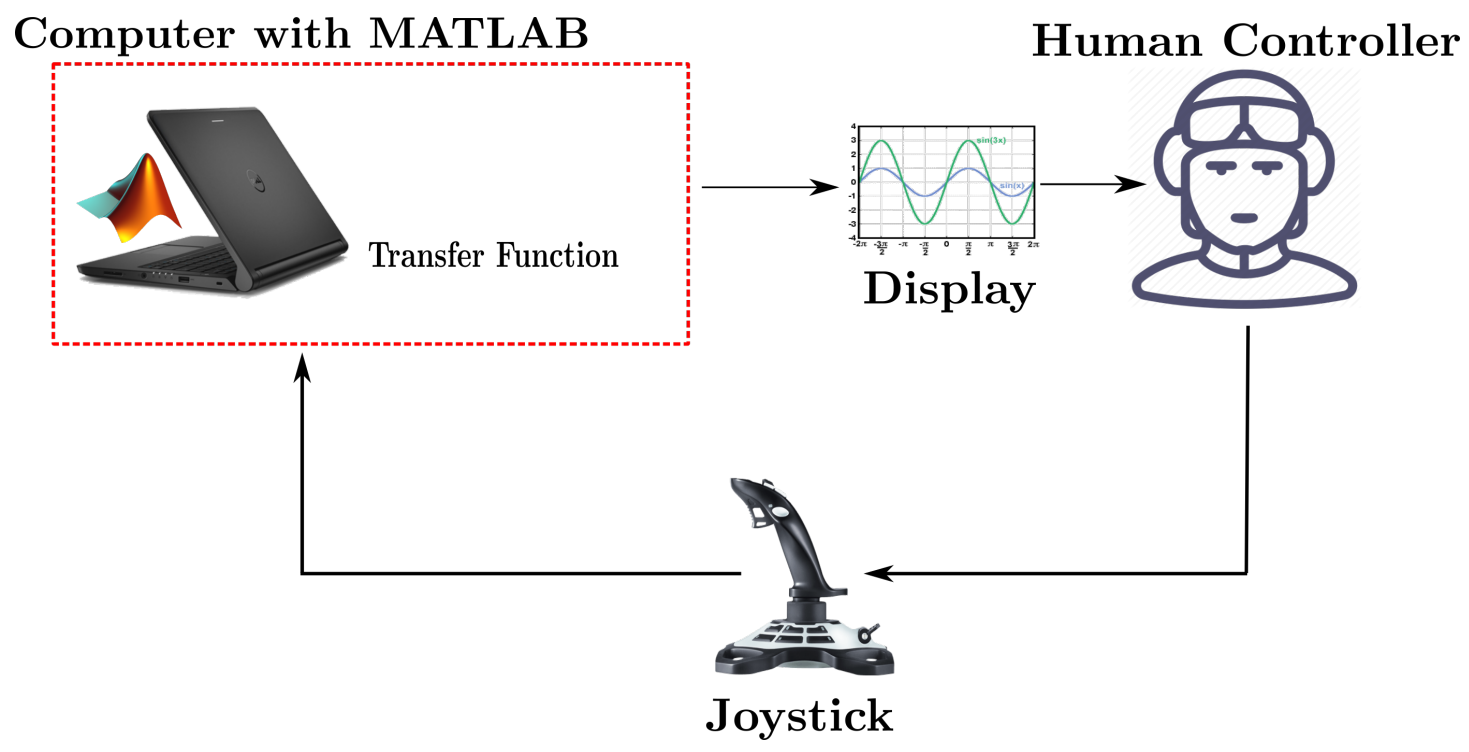

FIGURE 3.1: HuIL experiment setup.

subjects for nominal cases. Section 3.4 provides the estimation and stability prediction results for experiments performed with injected time delay, while Section 3.5 provides the estimation prediction results for the experiments performed with rate limiting Section 3.5. Section 3.7 presents the statistical analysis, while Section 3.8 provides a summary of the chapter.

\subsection{Description of HuIL Experiment}

This section provides a detailed description of the experimental setup and procedures used for the HuIL experiments. The only equipment needed for HuIL experiments are a computer that operates MATLAB and a joystick. For this study, an off-the-shelf gaming joystick (Logitech Extreme 3D pro) was used. The subjects who participated in the experiments used the joystick to perform a task displayed on the screen, and the joystick deflection measurement was considered as the human control output, which was used as input to the simulation. Further details are provided in the following sections.

\subsubsection{Experimental Setup}

Figure 3.1 shows the general flow diagram of the HuIL experiment setup. Due to the limited number of instruments needed, HuIL experiments are easy to set up and 
adapt to various scenarios. In HuIL experiments, the subject's control command was collected by the computer via USB connection. The joystick deflection command was then read by MATLAB and used to simulate the output using a pitch transfer function. The pitch error was calculated by subtracting the pitch output obtained from the desired pitch angle. The error was then displayed on a 21-inch monitor with the subject sitting approximately $0.5 \mathrm{~m}$ directly in front of the screen. Simulating a known transfer function ensures that the plant modeling errors do not affect the McRuer model parameter estimation or the closed-loop HCS stability prediction process and hence provides an accurate evaluation of the UKF algorithm.

To emulate a plant in the HuIL experiments, a typical third-order pitch transfer function representing the short period mode of a business jet was used[35], which is given by:

$$
\frac{\theta(s)}{\delta_{j}(s)}=\frac{2(s+0.3)}{s\left(s^{2}+0.65 s+2.15\right)}
$$

In Eqn. 3.1, $\theta(s)$ is the pitch output and $\delta_{j}(s)$ is the joystick deflection. The pitch to elevator deflection transfer function given by Eqn. 3.1 is inherently stable, with a gain margin and phase margin of infinity and $29.5 \mathrm{deg}$, respectively. Figure 3.2 shows the Bode plot of the pitch to elevator deflection transfer function given by Eqn. 3.1.

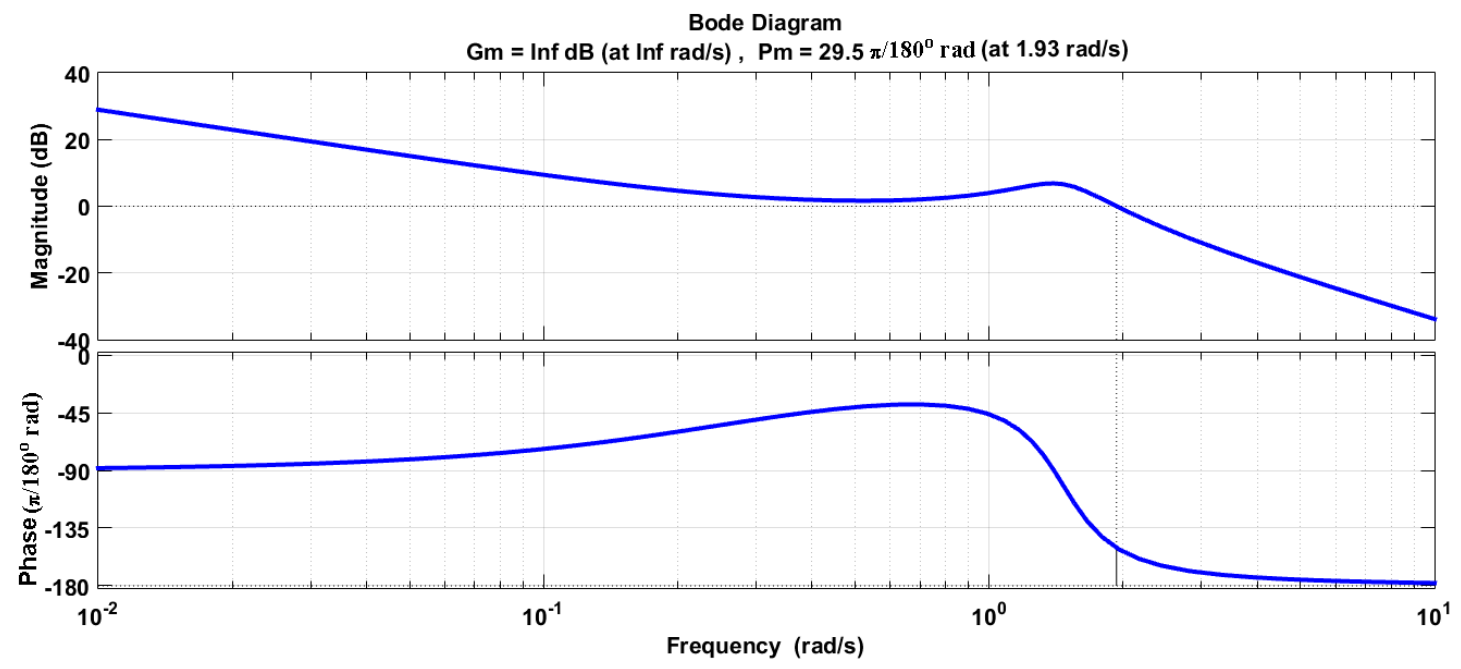

Figure 3.2: Bode plot of the pitch to joystick deflection transfer function (Eqn. 3.1) used for HuIL experiments. 
A latency analysis was performed to completely characterize the hardware and software used in the experiment. The latency between the joystick deflection and MATLAB interface to read the data was approximately $0.1 \mathrm{~s}$, determined using the joystick datasheet and MATLAB specifications on interfacing with external USB devices. The joystick deflection was scaled to +15 to $-15 \mathrm{deg}$, and the format of the display used to show the subject the error is shown in Figure 3.3. In Figure 3.3, the error is dis-

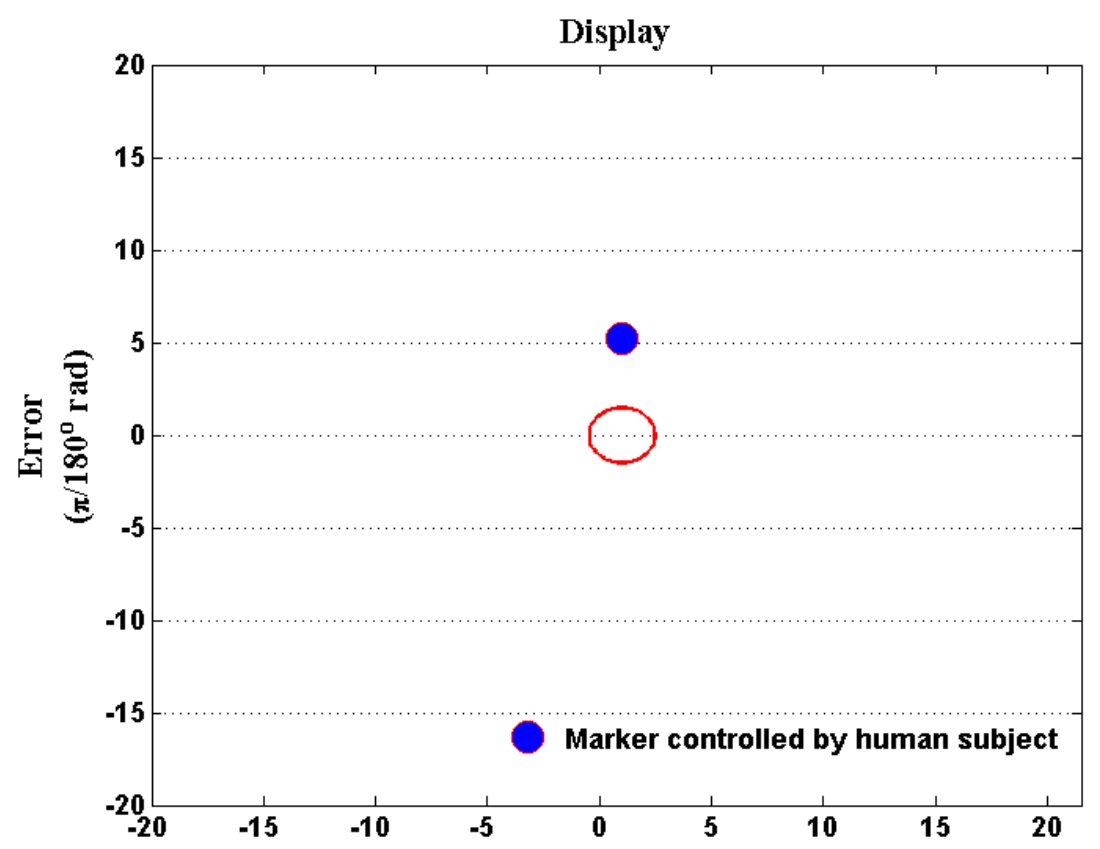

FigurE 3.3: Error display as seen by subjects.

played as the vertical displacement from the red circle centered at $(0,0)$. The subject was tasked with keeping the blue marker inside the red circle. In this study, only singleinput single-output tasks were investigated; thus, subjects were asked to control only the vertical displacement of the blue marker using the joystick. To investigate the human compensatory control behavior, the goal of the task was to minimize the pitch error.

The forcing function (i.e., desired pitch angle) used to calculate the pitch error was a sum of sines, with both low and high frequency components given by the following equation:

$$
f_{t}(t)=\Sigma_{k=1}^{N_{t}} A_{t}(k) \sin \left[\omega_{t}(k) t+\phi_{t}(k)\right]
$$

In Eqn. 3.2, $N_{t}=7$ (sufficient to prevent the subject from memorizing the pattern of the desired input $[28,29])$ was the number of sine waves, and $\omega_{t}, A_{t}$, and $\phi_{t}$ were the 
frequency, amplitude, and phase shift of the $k^{t h}$ sine wave, respectively. The measurement time used to construct the input was set to $T_{m}=101.63 \mathrm{~s}$, as this duration was found to be sufficiently long to enable estimator convergence, negate the initial condition effects, and generate sufficient data points for each subjects. The values of $\omega_{t}(k)$ were all integer multiples of the measurement time base frequency, $\omega_{m}=\frac{2 \pi}{T_{m}}=0.062 \mathrm{rad} / \mathrm{s}$ and were within the frequency range of the human control $(0.1-20 \mathrm{rad} / \mathrm{s}[2])$. Table 3.1 provides the properties of the input signal used, whereby all the phase shift values were randomly selected. In this study, frequency components were concentrated around a lower frequency range because it was found that, for the system described in Eqn. 3.1, at higher frequencies of input signal, the subjects were unable to perform the error reduction task with sufficient accuracy (small error). This also ensured that a major portion of human control output was linearly correlated with pitch error. The amplitude of the signal was selected to provide the subjects with sufficiently high perceptible error values.

TABLE 3.1: Properties of desired pitch angle (sum of sines).

\begin{tabular}{ccccc}
\hline $\mathbf{k}$ & $\mathbf{n}_{\mathbf{t}}$ & $\omega_{\mathbf{t}}, \mathbf{r a d} / \mathbf{s}$ & $\mathbf{A}_{\mathbf{t}}, \mathbf{d e g}$ & $\phi_{\mathbf{t}}, \mathbf{r a d}$ \\
\hline $\mathbf{1}$ & 11 & 0.68 & 1.8 & -0.5 \\
$\mathbf{2}$ & 7 & 0.43 & 1.8 & 1.06 \\
$\mathbf{3}$ & 12 & 0.74 & 1.8 & 2.06 \\
$\mathbf{4}$ & 15 & 0.93 & 1.8 & -0.57 \\
$\mathbf{5}$ & 9 & 0.56 & 1.8 & -4.51 \\
$\mathbf{6}$ & 10 & 0.62 & 0.18 & 0.13 \\
$\mathbf{7}$ & 32 & 1.98 & 0.18 & -1.22 \\
\hline
\end{tabular}

Figure 3.4 shows the sum of sines input corresponding to the values in Table 3.1 used for the HuIL experiments. The advantages of using sum of sines as a forcing function are summarized as follows:

1. Helps excite different frequency components in human control output.

2. Ensures that the estimator does not have observability issues.

3. Makes it difficult for the subject to predict or memorize the pattern; thus, the control output is predominantly compensatory. 


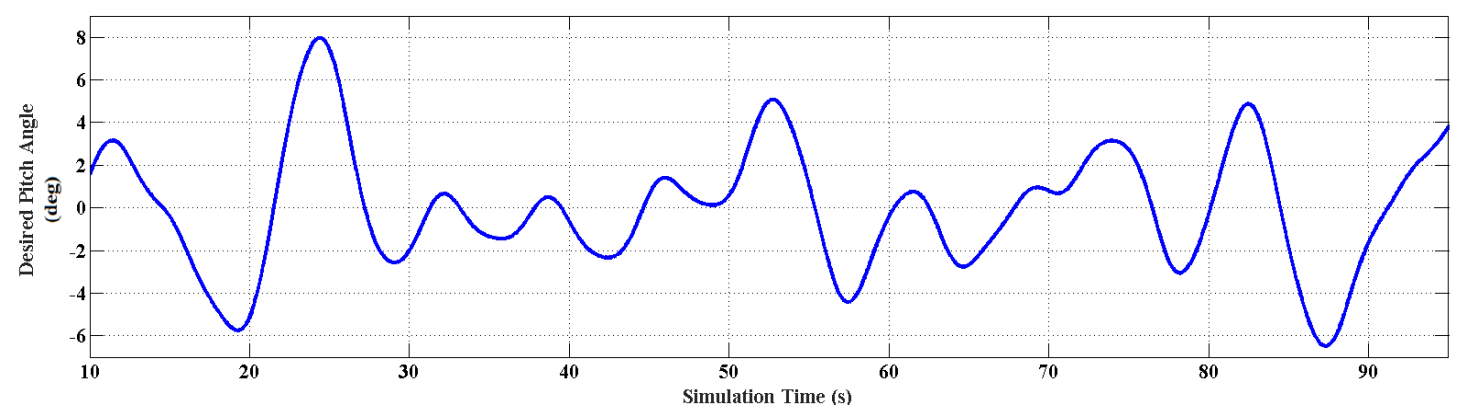

Figure 3.4: Typical sum of sines signal used as desired pitch angle.

4. Encourages the subject to have a low response threshold for the error ( i.e., a small dead band). Dead band is defined as the value of pitch error below which the subject does not generate any control commands (example data are shown in Figure 3.5 and Figure 3.6). This minimizes non-linear effects

Figure 3.5 shows an example of human control input and corresponding error for the input shown in Figure 3.4. The mean error and standard deviation of the pitch error



FigURE 3.5: Pitch error and joystick input for subject 1.

for Figure 3.5 were $-0.018 \mathrm{deg}$ and $0.74 \mathrm{deg}$, respectively. It can be seen from Figure 3.5 that the dead band was nearly nonexistent in the human control command. Figure 3.6 shows another example of the human control output and corresponding error for the input shown in Figure 3.4 .

The control command of subject 2 (Figure 3.5) to reduce pitch error was different to that of subject 1 (Figure 3.6), and subject 2 had some dead band. However, it can be seen that the error dead band was small and only occurred for a small percentage of the total experiment time ( $\sim 2 \%$ of the total time). The mean error and standard deviation of the pitch error for Figure 3.6 were $0.03 \mathrm{deg}$ and $0.97 \mathrm{deg}$, respectively. These figures 


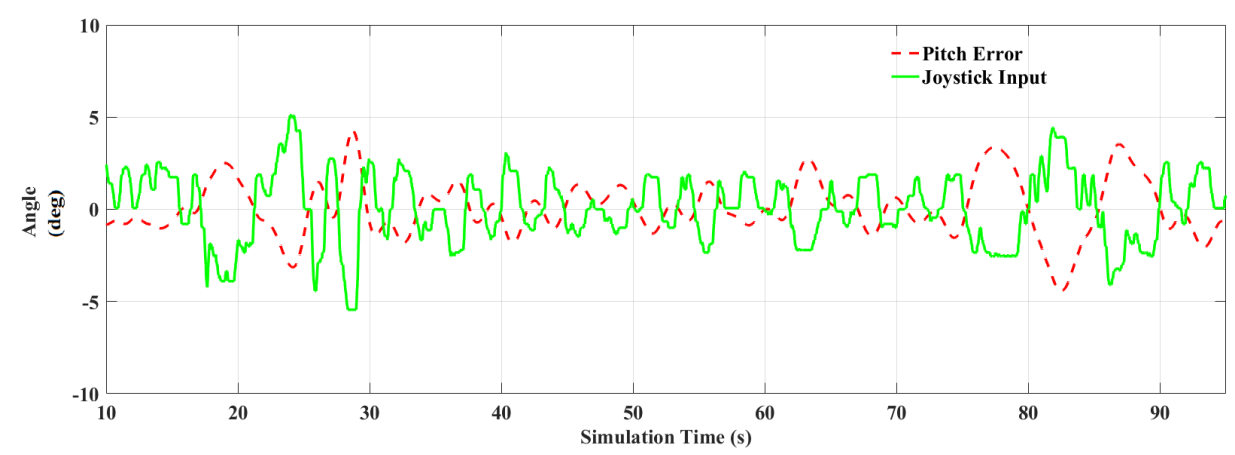

FiguRE 3.6: Pitch error and joystick input for subject 2.

provided further motivation to design an estimator capable of monitoring human control behavior in real-time as parameters of an assumed human control model change from one human controller to another.

\subsubsection{Study Procedure}

It was important that the HuIL experiments were performed properly to ensure the quality of the data used to validate the estimator. The following protocol was submitted to the WVU Institutional Review Board (IRB) for review. The IRB approval for this experimental procedure is on file and is provided in A.

For this study, 11 volunteer subjects (10 males and one female) were recruited, aged between 20 to 35 years old. A post-hoc statistical analysis was carried out to determine the statistical power of the results and is presented in Section 3.7. All subjects had previous experience using a joystick to play video games. At the beginning of the experiment, each subject was given a detailed explanation of the HuIL experiments and the task (i.e., reducing the pitch error) to be performed by them. They were asked to sit in front of the screen that displayed the pitch error, as shown in Figure 3.3, and were given instructions on how to use the joystick to perform the task. For training, each subject was given five trials to become accustomed to the experiment. Following training, each subject was given four additional trials to attain a baseline performance. The baseline was then used to compare the estimator results from three trials each for delay injection in the control loop and rate limiting of the joystick command. There was no required minimum time gap between each group of experiments as long as the 
subject felt comfortable performing the experiments. The entire experiment typically lasted between 30 and 45 minutes. Post-trial, the value of the mean error and standard deviation of the pitch error were checked to ensure task completion integrity. The IRB approval for this experiment is provided in Appendix A.

The data from the training trials provided insights into the change of control behavior of the subjects as they improved in performing the task. As each subject underwent training, it was observed that the standard deviation of the pitch error decreased with successive trials; however, the rate of decrease was not the same between different subjects. In addition, the minimum standard deviation of pitch error achieved by different subjects was found to differ. This again emphasizes the variable nature of human control behavior. Figure 3.7 shows the change in standard deviation of the pitch error with respect to successive trials for five different subjects.

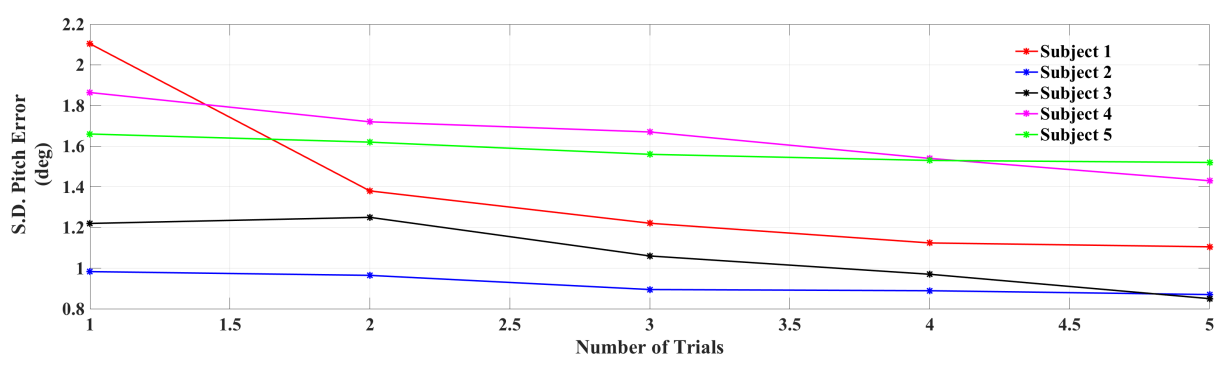

FIGURE 3.7: Change in the standard deviation of the pitch error with successive trials.

Post training, subjects carried out the same task with the injection of time delay and rate limiting in the control loop. Time delay values of $100 \mathrm{~ms}, 200 \mathrm{~ms}$, and $300 \mathrm{~ms}$ were used. During the delay experiments, joystick commands were obtained using the following equation:

$$
P(k)=P\left(k-\frac{\tau_{i}}{\delta t}\right)
$$

Where $\tau_{i}$ is the time delay injected in seconds, $\delta t$ is the sampling time in seconds, and $P(k)$ is the joystick command at time step $k$ at time $k \times \delta t$. In all HuIL experiments with delay injection, $\tau_{i}$ was an integer multiple of $\delta t$. In addition, the time instant at which the delay was injected in the system was randomly selected and not known to the subject. Therefore, the subject performing the task had no knowledge of the amount of delay injected or the time of delay injection in the system, and subsequently could 


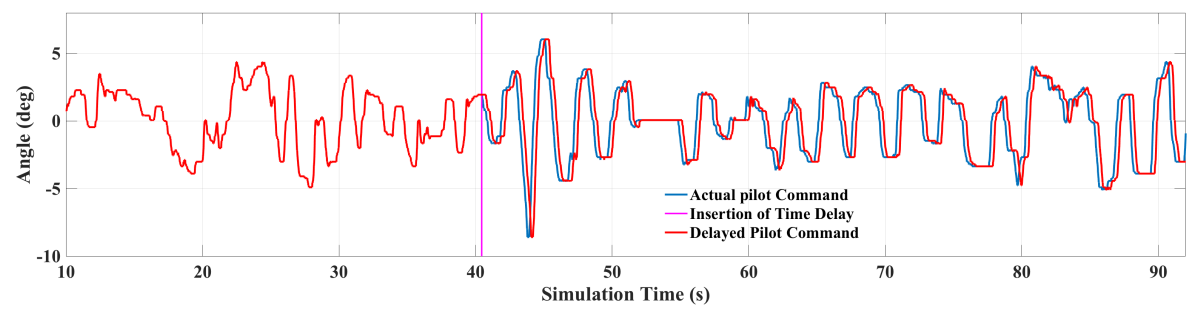

FiguRE 3.8: Delayed (200 ms delay) joystick command HuIL experiment.

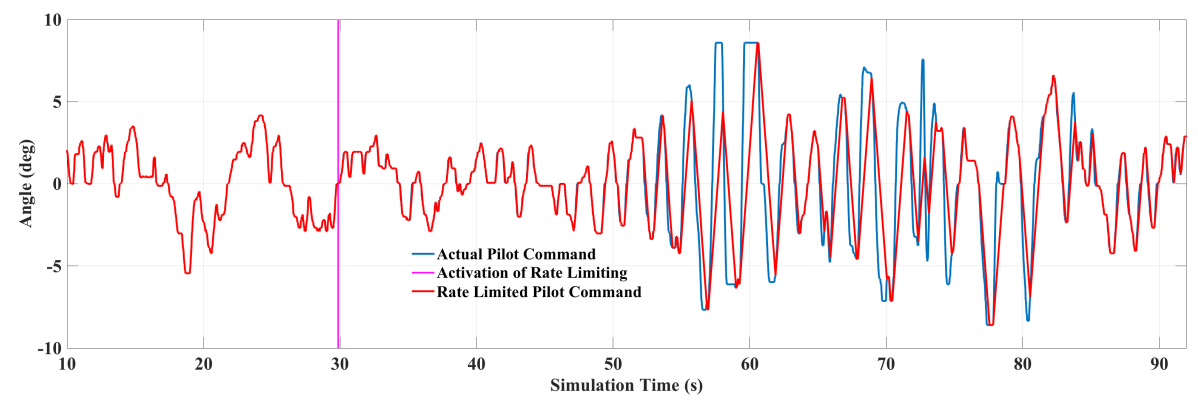

Figure 3.9: Rate limited (20 deg/sec) joystick command.

not attempt any preemptive action. To increase the sense of urgency in a few randomly selected experiments, a step disturbance (with amplitude of 3 deg) was added to the pitch output at the time of introducing the time delay in the system. Figure 3.8 shows an example dataset of the delayed joystick command and actual joystick command.

For rate-limiting trials, the joystick commands were rate limited using the following equation:

$$
P(k)= \begin{cases}P(k-1)+\operatorname{sgn}(P(k)-P(k-1)) \times R_{L} \times \delta t, & \frac{|P(k)-P(k-1)|}{\delta t}>R_{L} \\ P(k), & \frac{|P(k)-P(k-1)|}{\delta t}<R_{L}\end{cases}
$$

Where $R_{L}$ is the maximum permitted rate of joystick deflection. For HuIL experiments, rate-limiting values of $30 \mathrm{deg} / \mathrm{s}, 20 \mathrm{deg} / \mathrm{s}$, and $10 \mathrm{deg} / \mathrm{s}$ were used. Similar to the delay experiments, the subjects had no knowledge of the magnitude of the rate limit or the time at which the rate limit was first activated during the experiments. Similarly, in a few randomly selected experiments, a step disturbance was added at the time of activating the rate limiting in the system. Figure 3.9 shows an example dataset of rate-limited joystick command and actual joystick command. 
The joystick and pitch error data collected for different trials were used with the UKF algorithm to estimate the McRuer model parameters as described in the following sections.

\subsection{McRuer Model Parameter Estimation}

The parameter estimation effort used the procedure described in the previous chapter (Section 2.2). The estimated parameters were then used to make real-time stability predictions about the closed-loop system, which were then compared with the actual experimental data. The noise matrices (i.e., process noise, $\mathrm{Q}$, and measurement noise, R) for the UKF algorithm were tuned using the Monte Carlo technique to ensure optimal estimator performance. In addition, the initial condition used for all UKF estimation was given by the following:

$$
x_{0}=\left[\begin{array}{llllllll}
10.50 & 20 & -0.43 & 2.11 & 6 & 10.80 & 0 & 0
\end{array}\right]^{T}
$$

The above initial state was obtained using Monte Carlo simulations and other similar studies of the McRuer model[2, 17]. The values in Eqn. 3.5 correspond to $K_{\text {pilot }}=$ $0.54, T_{\text {Lead }}=0.32, T_{\text {Lag }}=0.4$, and $\tau=0.25 \mathrm{~s}$. As described in the previous chapter (Section 2.9), the input to the estimator was the pitch error displayed on the screen to the subject, and the subject's joystick commands. The outputs of the estimator were the McRuer model parameters and the filtered subject's state. Figure 3.10 displays a set of typical pitch error and joystick command values obtained from a normal HuIL experiment (i.e., no delay or no rate limiting) used in the estimation process.

Figure 3.10 also shows the plot of pitch rate with time, as pitch rate provides more intuitive information regarding the presence of oscillation or divergence in closed-loop HCS (in this case, closed-loop pitch control task in HuIL experiments). The pitch angle can have a non-zero mean and small value at the initiation phase of an unfavorable human-machine interaction, making it difficult to detect motion and oscillation, which is not the case with the pitch rate. It can be seen from Figure 3.10 that the HCS does 


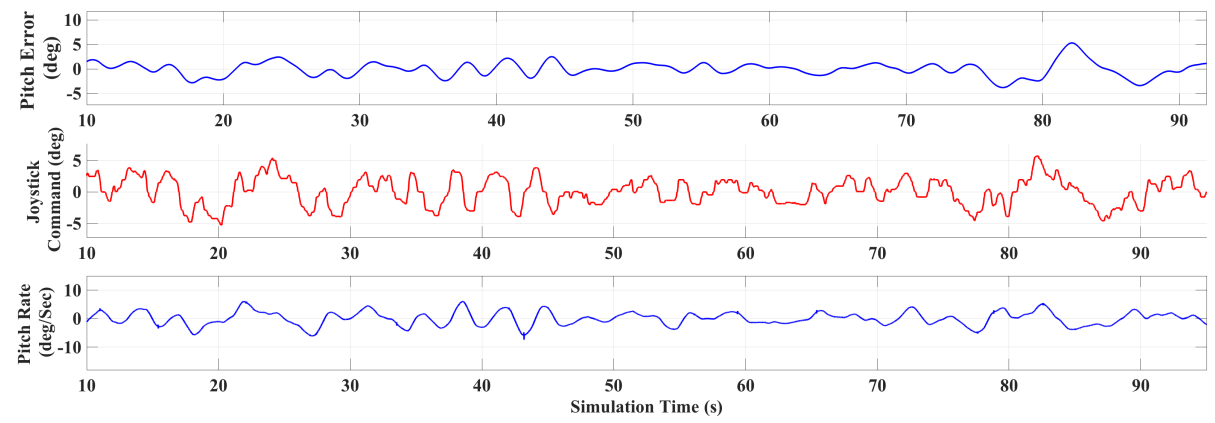

Figure 3.10: Typical set of data used for estimation under nominal condition (no delay injection or rate limiting of joystick commands).
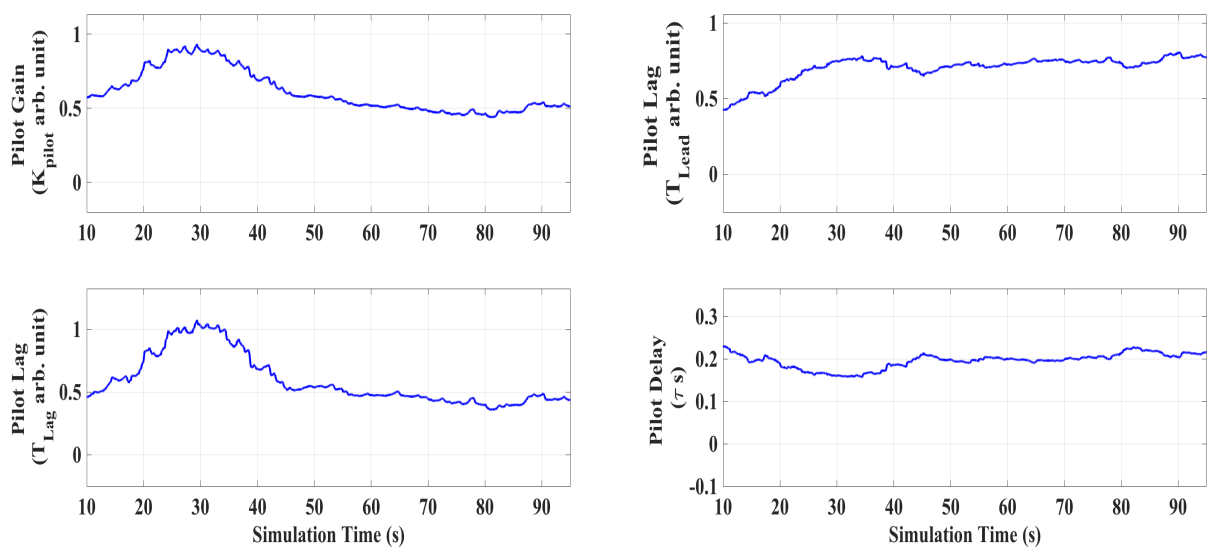

FiguRE 3.11: Typical set of data used for estimation under nominal condition (no delay injection of rate limiting of joystick commands).

not show any unfavorable oscillating tendency or instabilities for this particular HuIL experiment.

The pitch error data and subject's joystick command shown in Figure 3.10 were used in the UKF algorithm to estimate the McRuer model parameters for the subject. The estimated parameters are shown in Figure 3.11.

It can be observed from Figure 3.10 that the subject was most active between 15 to $45 \mathrm{~s}$ (standard deviation of joystick command: $3.03 \mathrm{deg}$ ) and 78 to $92 \mathrm{~s}$ (standard deviation of joystick command: $1.96 \mathrm{deg}$ ), compared to the time interval from 45 to $78 \mathrm{~s}$ (standard deviation of joystick command: $0.82 \mathrm{deg}$ ), which was expected due to the design of forcing function. In Figure 3.11, it can be seen that the McRuer model gain and lag parameters show significant changes in the time interval 15-45 s, which correlated with the subject trying to reduce large variations in pitch error during these 


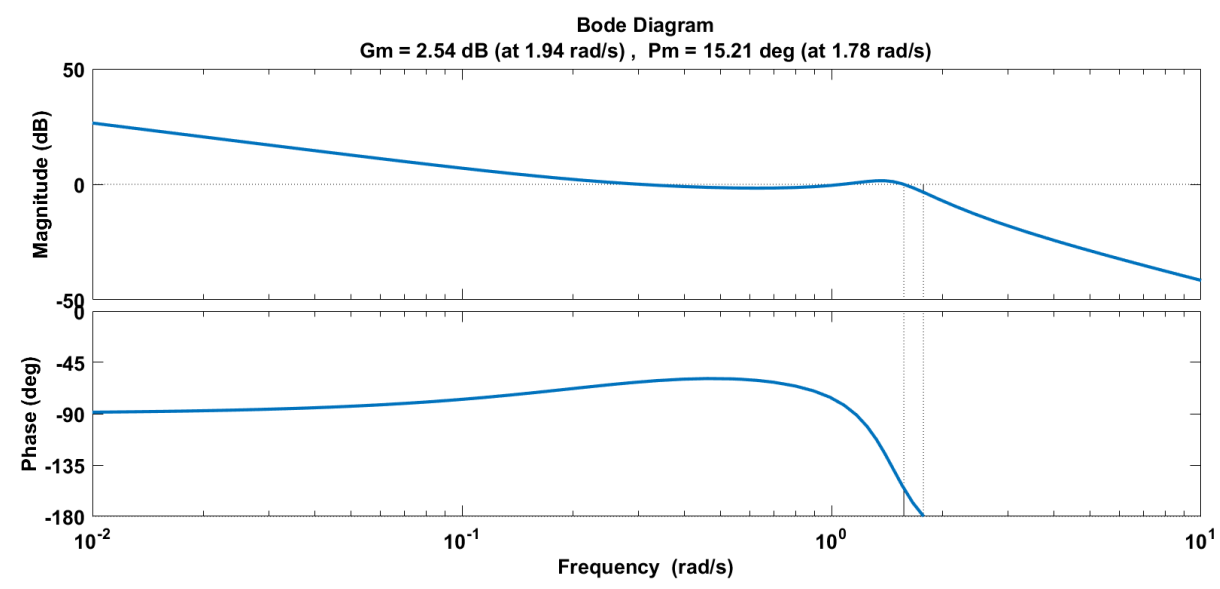

Figure 3.12: Bode plot of the HCS at 35s after the start of the experiment.

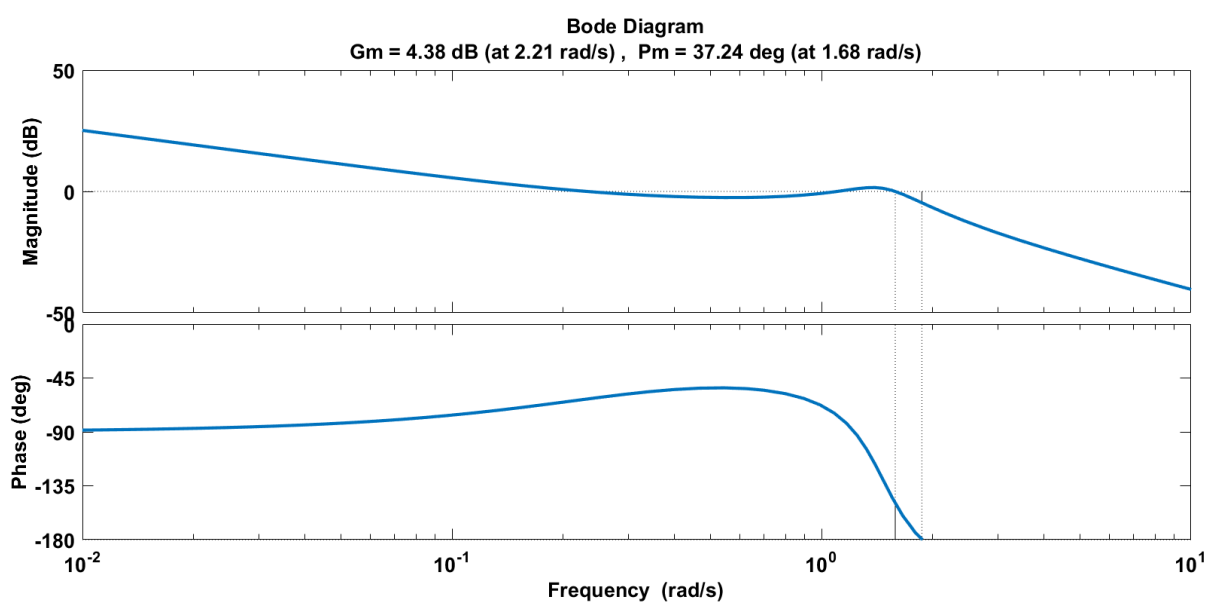

Figure 3.13: Bode plot of the HCS at 65s after the start of the experiment.

time intervals, as seen in Figure 3.10. Conventional batch estimation of McRuer model parameters would not be able to capture such variations, which may contain important information about the performance of the closed-loop HCS. Figures 3.12, 3.13, and 3.14 show the Bode plot of the open-loop HCS system at 35, 65, and $90 \mathrm{~s}$, within the interval 15 to $45 \mathrm{~s}, 45$ to $78 \mathrm{~s}$, and 78 to $92 \mathrm{~s}$.

From the Bode plots in Figures 3.12, 3.13, 3.14 it can be seen that the open-loop HCS had a positive gain margin for the McRuer model parameters estimated at 35, 65, and $90 \mathrm{~s}$. It is interesting to note that the margins at $35 \mathrm{~s}$ and $90 \mathrm{~s}$ were lower than the margins at $65 \mathrm{~s}$, which corresponds to the earlier observations regarding joystick activity during these time intervals. For this particular subject, the smaller values of margins at $35 \mathrm{~s}$ may be attributed to the subject becoming familiar with the control task at the beginning of the experiment. In addition, the value of the gain crossover frequency at 


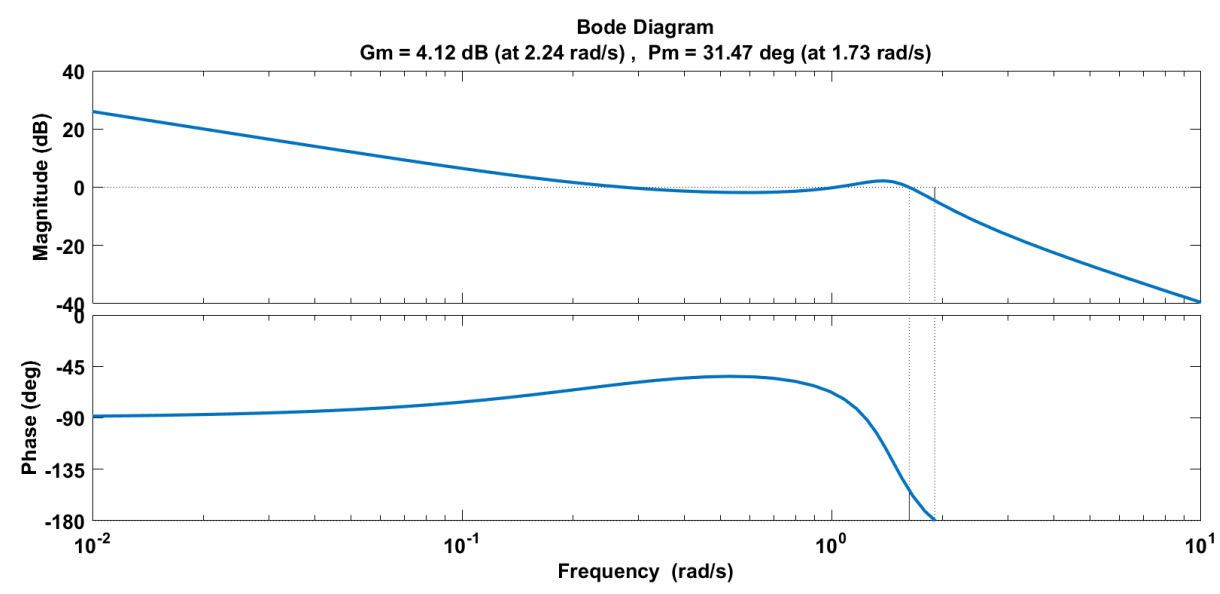

Figure 3.14: Bode plot of the HCS at 90s after the start of the experiment.

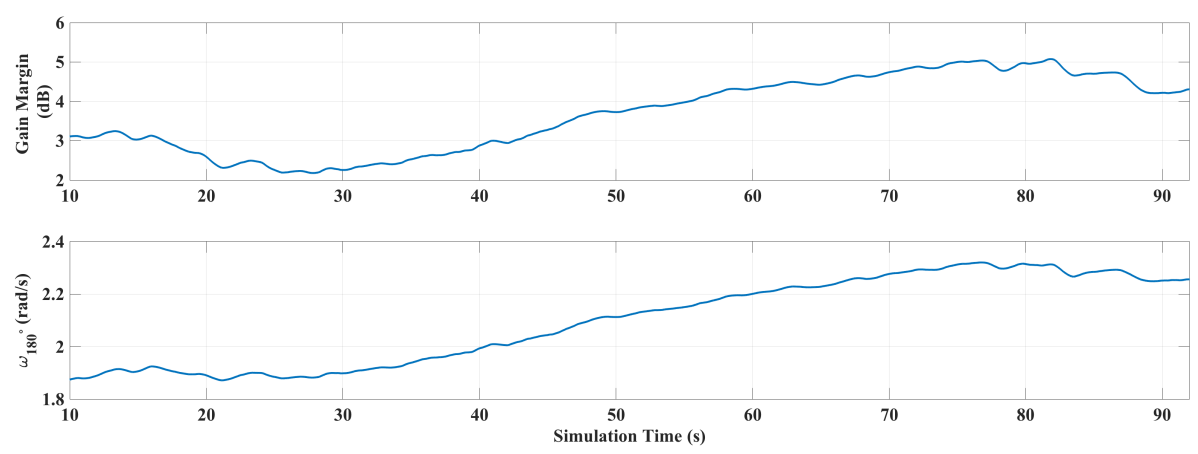

FiguRE 3.15: Change in gain margin and phase cross over frequency with time.
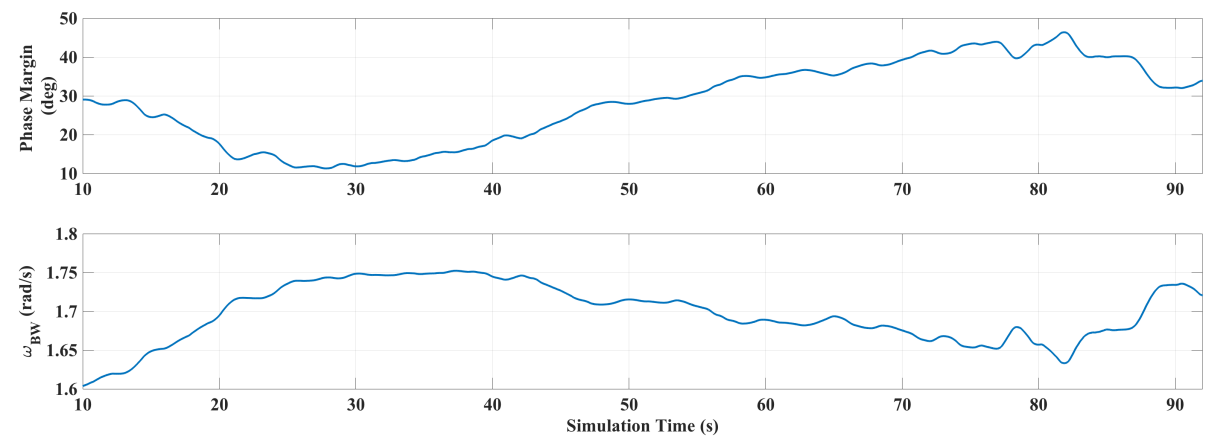

FiguRE 3.16: Change in phase margin and gain cross over frequency with time.

35,70 , and $90 \mathrm{~s}$ were $1.78,1.68$, and $1.73 \mathrm{rad} / \mathrm{s}$, respectively, which were close in value. Similar values of the gain crossover frequency suggest that the subject was adapting to obtain similar task bandwidth. Similar margin calculations were performed over the entire duration of the experiment. Figures 3.15 and 3.16 show the change in margins with simulation time and corresponding crossover frequencies.

The change in the margins with time correlated with the observations made from 
Figure 3.11. At the beginning (15-45 s), the subject was becoming familiar with the task and working to reduce large errors; thus, the subject can be thought to have a slightly higher bandwidth (i.e., to have higher gain range) to accomplish the task, and hence lower gain and phase margin. For the interval of 45 to $78 \mathrm{~s}$, the error values were small and the subject did not require a large bandwidth to perform the task. Hence, as can be seen from Figures 3.15 and 3.16, the HCS had a smaller bandwidth, yet larger gain and phase margin. Similarly, in the time interval of 78 to $92 \mathrm{~s}$, the error values were again large and the subject increased the bandwidth to perform the task with sufficient accuracy and, in the process, reduced the gain and phase margin. In addition, to attain information regarding the asymptotic stability of the HCS obtained for each set of estimated McRuer model parameters, the locations of the closed-loop poles of the HCS were determined. However, to monitor the stability of the HCS in real-time, the HCS obtained at each time instant using the estimated parameters was assigned a stability metric valued based on the following equation:

$S_{k}= \begin{cases}1-\frac{0.5\left(20-P M_{k}\right)}{20}-\frac{0.5\left(3-G M_{k}\right)}{3}, & \text { All the closed loop HCS poles are in LHP } \\ 0, & \text { At least one of the closed loop HCS poles is in RHP }\end{cases}$

TABLE 3.2: Definition of stability metric prediction regions.

\begin{tabular}{ccc}
\hline Region Color & Stability Metric Range & Stability Prediction \\
\hline Red-HMO & {$\left[\begin{array}{ll}-\infty & 0.25\end{array}\right]$} & High Probability of Instability \\
Orange-HMO Prone & {$\left[\begin{array}{lll}0.25 & 0.75\end{array}\right]$} & Median Probability of Instability \\
Green-No HMO & {$\left[\begin{array}{ll}0.75 & \infty\end{array}\right]$} & Low Probability of Instability \\
\hline
\end{tabular}

In the above equation, $S_{k}$ is the stability metric value at time step $k, G M_{k}$ is the gain margin of the HCS at time step $k$, and $P M_{k}$ is the phase margin of the HCS at time step $k$. It was observed from all HuIL experiments that the subjects did not show any difficulty in performing the pitch error reduction task with sufficient accuracy if the gain margin was at least $3 \mathrm{~dB}$ and the phase margin was at least $20 \mathrm{deg}$. Therefore, Eqn. 3.6 used $3 \mathrm{~dB}$ and $20 \mathrm{deg}$ as a boundary condition, along with the information regarding 


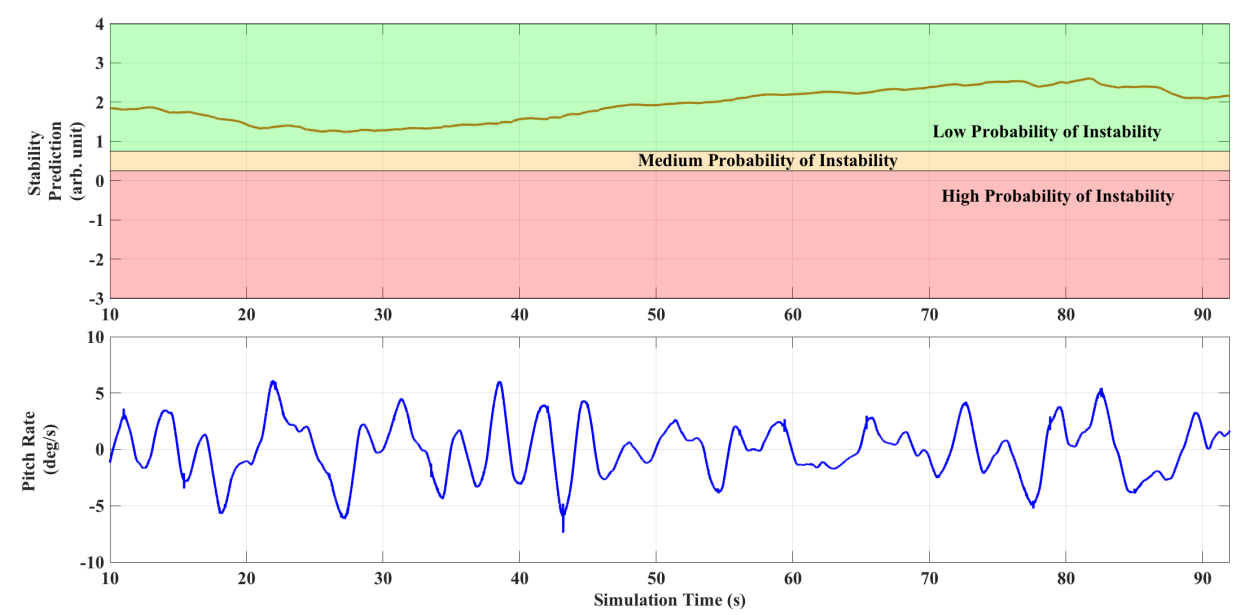

FiguRE 3.17: Stability metric value of the closed-loop HCS with time.

the locations of the closed-loop poles of the HCS to assign a stability metric value to the HCS at time step $k$. From Eqn. 3.6, it is immediately apparent that a HCS with higher value of $S_{k}$ is more stable (higher values of margins) and hence less likely to produce human machine oscillation (HMO) or become unstable compared to a HCS with smaller value of $S_{k}$ (lower values of margins). Figure 3.17 shows the stability metric value of the closed-loop HCS with time for the experiment presented in Figure 3.10.

In Figure 3.17, different HCS regions are shown, which are defined in Table 3.2. The ranges in Table 3.2 were obtained by analyzing the experimental data and subjects' description of the task difficulty. It is clear from Figure 3.17 that the HCS stability value predominantly lies in the green (i.e., no HMO) region which was supported by the lack of undesirable oscillations the pitch rate plot in Figure 3.17.

A similar analysis was undertaken for all the subjects involved in the experiments. Figure 3.18 shows the estimation results for five different subjects under the same input forcing function under nominal condition (i.e., no time delay injection and no joystick command rate limiting). As shown in Figure 3.18, different subjects' control behavior varied, yet still showed similar trends due to the control task and input forcing function being the same for all the HuIL experiments. As with earlier cases, Figures 3.19 and 3.20 show the variation in margins and crossover frequencies with time for the same five subjects shown in Figure 3.18. 

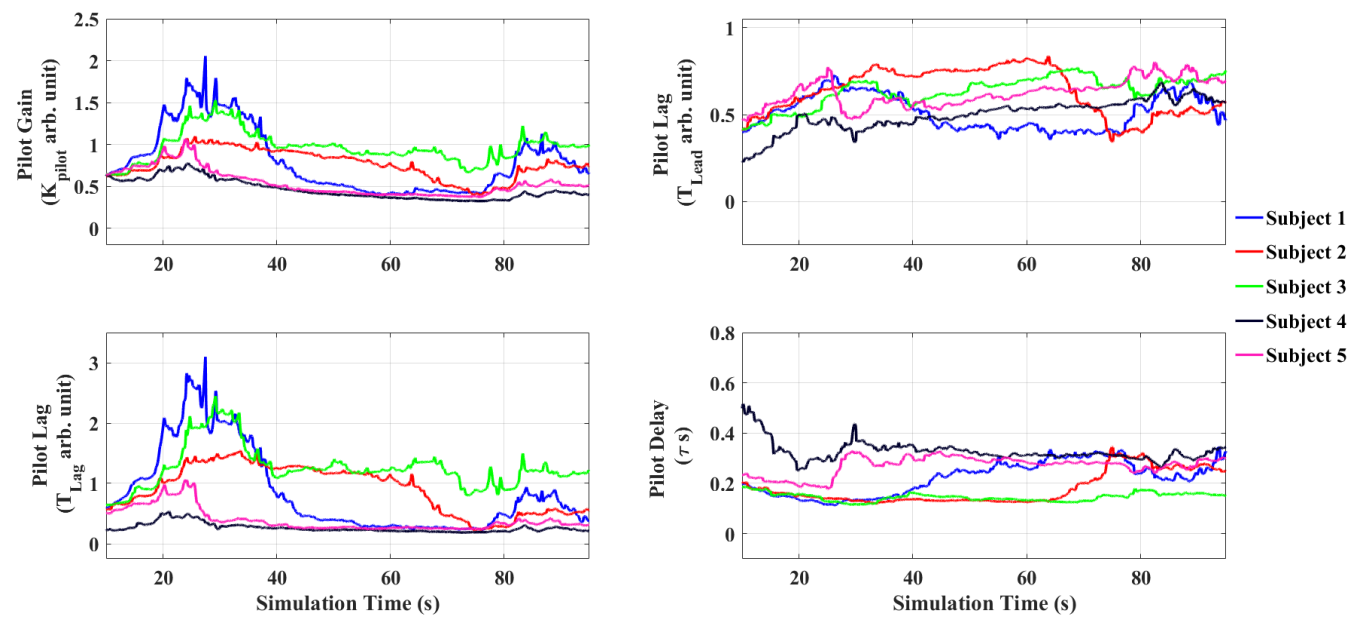

FIGURE 3.18: Comparison of estimated parameters for 5 different subjects for a single trial under nominal condition.
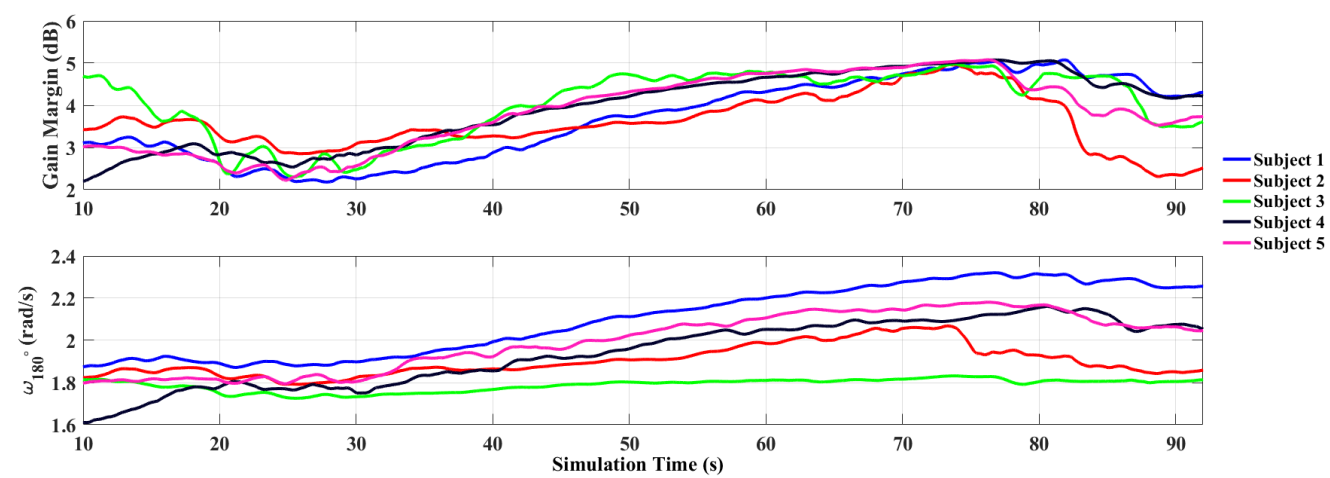

FIGURE 3.19: Variation in gain margin and phase crossover frequency for same 5 different subjects.

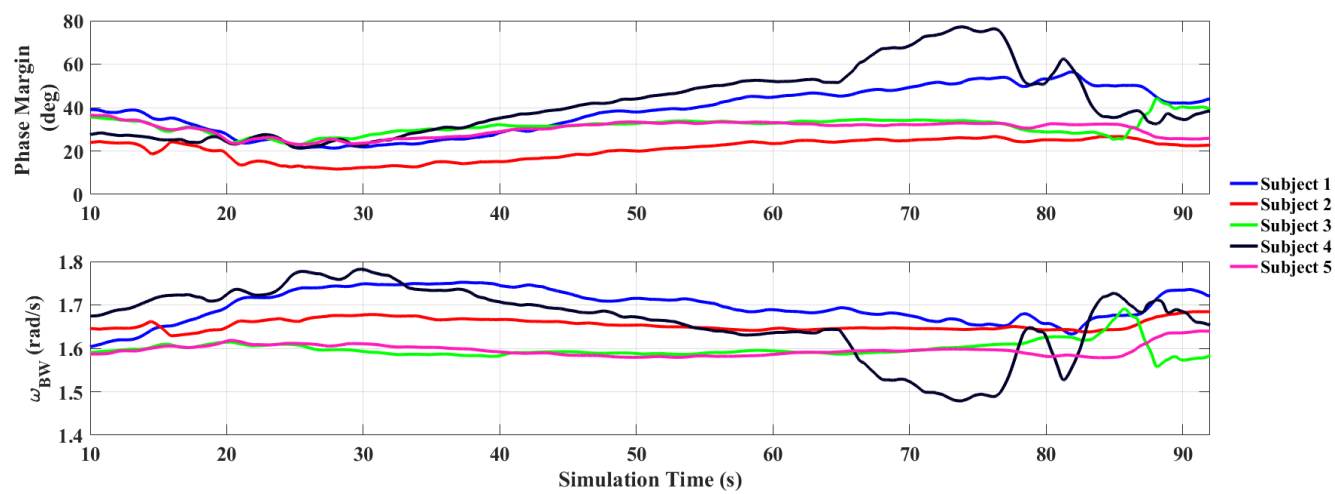

Figure 3.20: Variation in gain margin and phase crossover frequency for same 5 different subjects. 
Similar to the estimated McRuer model parameters, the variations in margins and crossover frequencies followed a similar trend for different subjects under nominal conditions, as explained in the earlier parts of this section. Further, it was found that the closed-loop HCS stability metric value was predominantly in the green region (i.e., no HMO) for all the subjects for the nominal case, which was validated by the lack of undesirable oscillations in the pitch rate data for these HuIL experiments. The analysis performed for various subjects under nominal conditions was used to compare the subject performance when the HuIL experiments were undertaken with injected time delay or rate limiting described in the following two sections.

\subsection{Estimation Results for HuIL Experiments with Time Delay}

The purpose of the HuIL experiment was to evaluate the ability of the McRuer model constructed with the estimated model parameters to predict the stability of the closed-loop HCS under both nominal and off-nominal conditions in real-time. For HuIL experiments with an injected time delay, the UKF estimation was undertaken with pitch error and actual joystick command (i.e., the joystick command before it was delayed artificially). The parameters obtained were then used to obtain the stability characteristics of the closed-loop HCS, and the stability predictions were then compared with the actual data.

Figure 3.21 shows a set of pitch angle, joystick command, and pitch rate values for a HuIL experiment with a 200 ms time delay used in the estimation process. The standard deviation of the pitch error before delay injection for the data shown in Figure 3.21 was found to be $0.87 \mathrm{deg}$, and after injection of time delay, the standard deviation of the pitch error was found to be $2.01 \mathrm{deg}$. Similarly, the standard deviation of the joystick command before insertion of time delay was found to be 2.27 deg and post-time delay injection, the standard deviation of the joystick command was found to be 3.49 deg. Therefore, it became more difficult for the subject to perform the task after the injection 

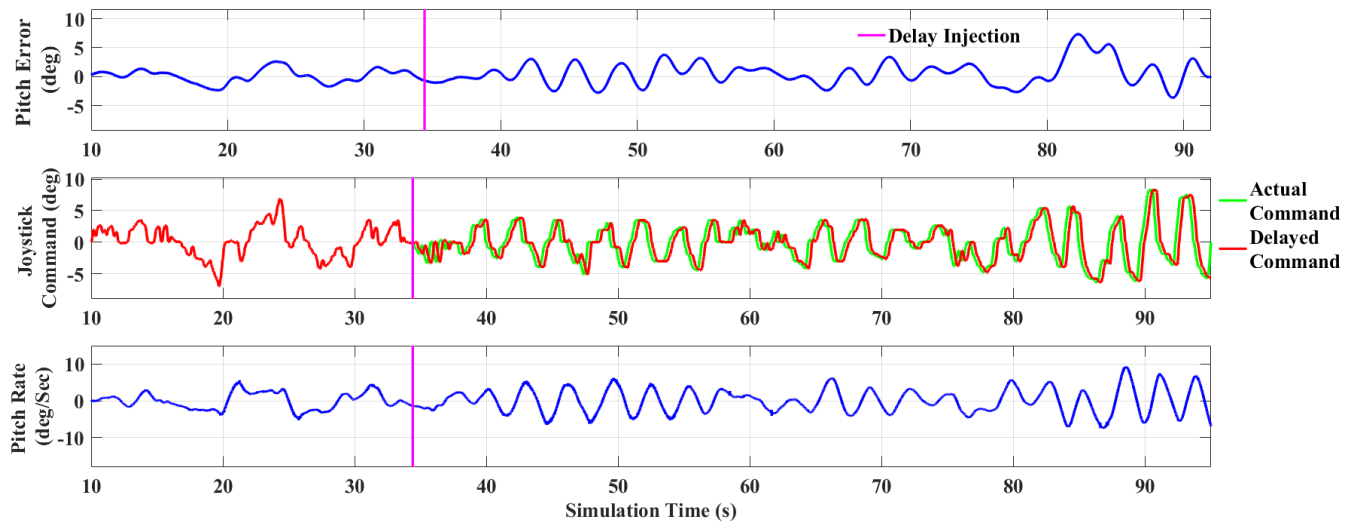

Figure 3.21: Pitch error, joystick command and pitch rate for the HuIL experiment with $200 \mathrm{~ms}$ time delay injected.
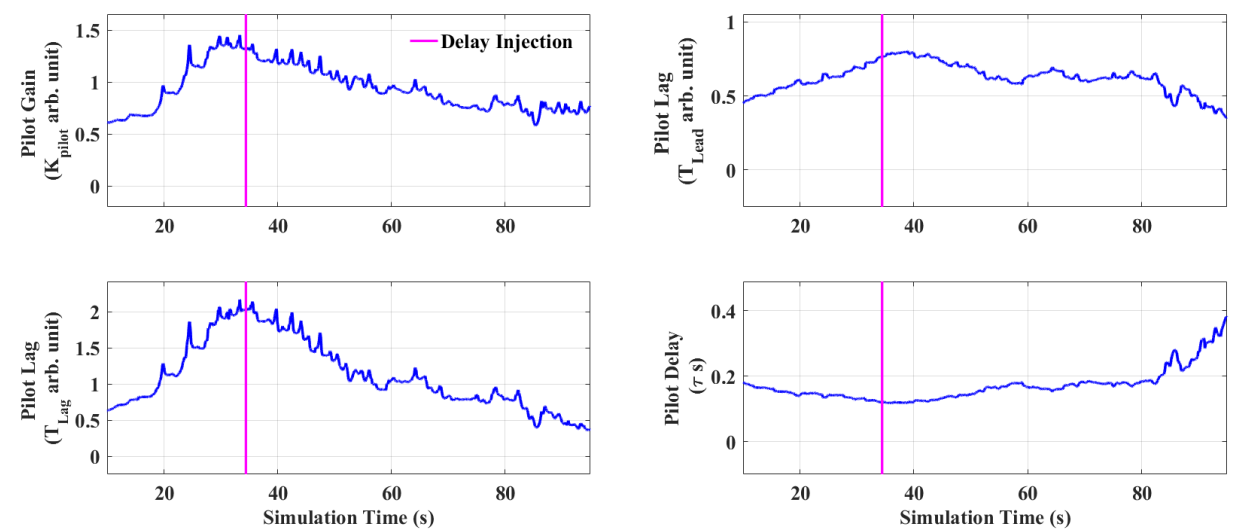

Figure 3.22: Estimation results for data shown in Figure 3.21.

of delay. In addition, it can be clearly seen that the HCS had oscillating tendencies post-injection of the time delay from the pitch rate plot in Figure 3.21.

To predict the stability of HCS, frequency analysis techniques such as the Bode plot can be used to calculate the value of stability metric using Eqn. 3.6. As before, the McRuer model was obtained using the parameters estimated in real-time from the UKF algorithm. Figure 3.22 shows the McRuer model parameters obtained for the data shown in Figure 3.21.

In Figure 3.22, after the injection of the time delay, it can be seen that the estimated delay $(\tau)$ gradually increased. This can be attributed to the subject waiting longer to understand how the pitch error was evolving with respect to the joystick command before moving the joystick. 

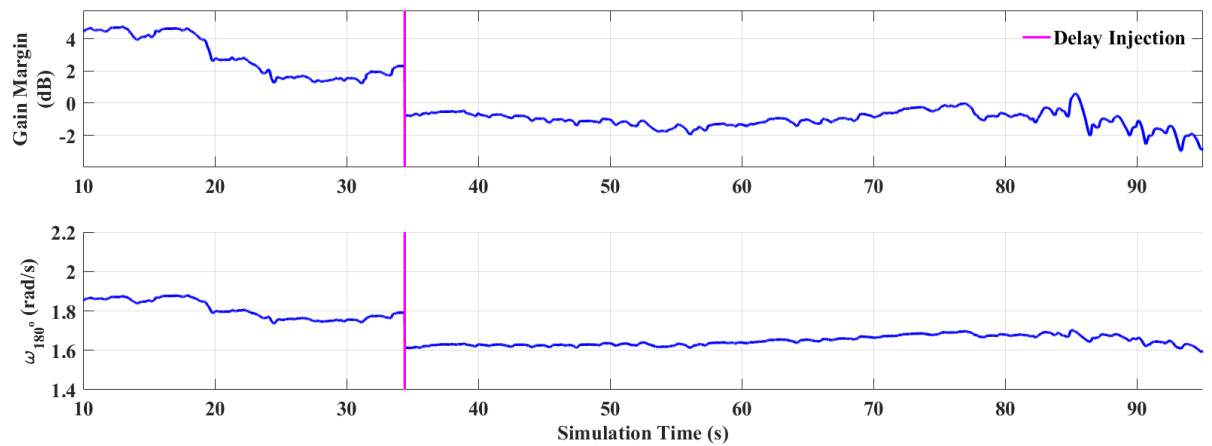

FIGURE 3.23: Change in gain margin and phase crossover frequency with time for experiment represented in Figure 3.21.
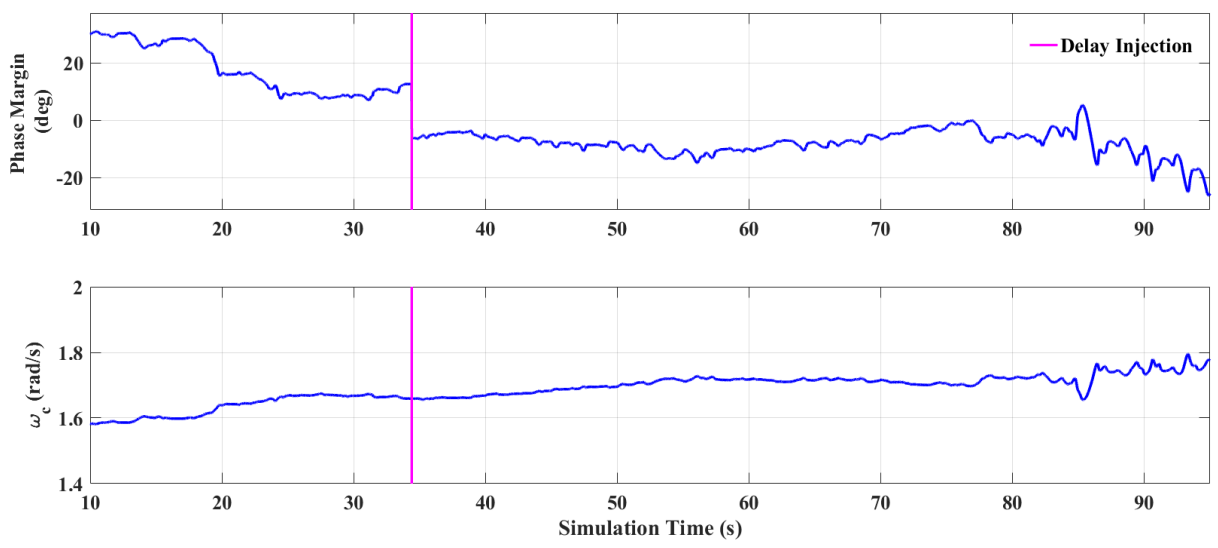

FIGURE 3.24: Change in phase margin and gain crossover frequency with time for experiment represented in Figure 3.21.

It is evident from the joystick command plot in Figure 3.21 that, post-delay injection, some portion of the joystick commands was stationary at values close to zero. It can also be seen from the parameter plot that the subject was unable to generate sufficient lead compared to the HuIL experiments without any delay injection. Similar to the earlier HuIL experiments under nominal conditions, the McRuer model obtained using the parameters in Figure 3.22 was used to calculate the change in margins and crossover frequency with time for the HuIL experiment represented in Figure 3.21.

It is apparent from the margins plot and phase crossover frequency plot in Figures 3.23 and 3.24 that there was a jump when the delay was first injected in the HuIL experiment. This jump can be attributed to the change in the system dynamics due to the addition of the time delay. However, the absence of a similar jump in the gain 



FigURE 3.25: Stability metric value of closed-loop HCS with time for HuIL experiment with $300 \mathrm{~ms}$ time delay injection (Figure 3.21).

crossover frequency suggests that the subject maintained a similar task bandwidth overall. However, there was a slight increase in the bandwidth post-injection of the time delay. Figure 3.25 shows the stability metric value of the closed-loop HCS with time for the HuIL experiment with time delay.

In Figure 3.25, the McRuer model (obtained using parameters estimated in real-time by the UKF algorithm) predicted instability (i.e., at least one closed-loop HCS pole was in the right-hand plane) post injection of time delay. In the same region, the pitch rate showed an increased amount of undesired oscillations. Therefore, it can be said that the McRuer model obtained using estimated parameters was able to determine the HCS stability characteristics, which agreed with the observations made using the pitch rate data.

The real-time prediction of stability (RPStab) method described above for evaluating the closed-loop HCS stability, needed to be validated, which required detection of oscillations in the experimental data. The RPStab prediction results were also compared with two separate real-time oscillation identification/detection algorithms/techniques: (i) real-time oscillation verifier (ROVER) [36] and (ii) windowed discrete Fourier transform (wDFT) [37]. The ROVER detection of oscillations uses the following four types of input:

1. Magnitude of the pitch rate $(q)$ - obtained by subtracting the recent local minima from the recent local maxima of the pitch rate data. 


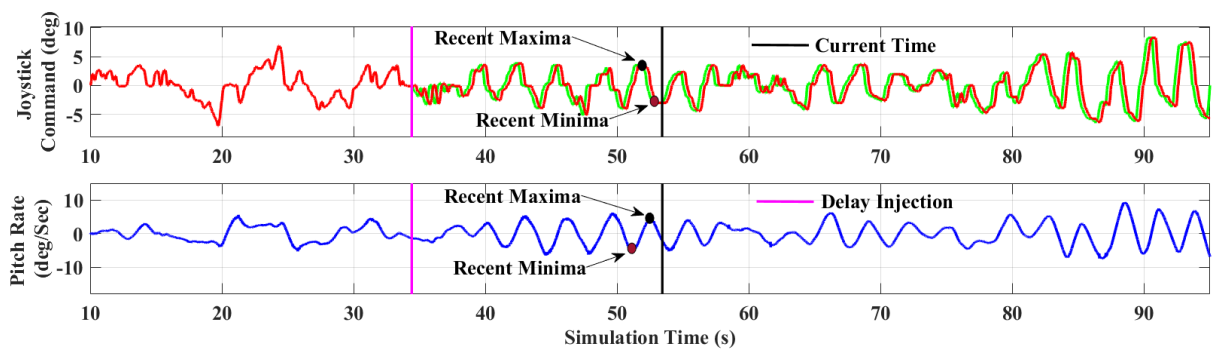

FiguRE 3.26: Recent minima and maxima selection for ROVER. Cyan vertical line represents time when delay was injected and black vertical line represents the current time till which ROVER analysis has been carried out.

2. Magnitude of the commanded elevator deflection (Joystick deflection in this case) $\left(J_{d}\right)$ - obtained by subtracting the recent local minima from the recent local maxima of joystick data.

3. Phase angle difference between the pitch rate and the joystick command $(\Delta \Phi)$ - obtained from the time difference between the recent local maxima of joystick command and recent local maxima of pitch rate.

4. Frequency of the pitch rate oscillations $\left(f_{q}\right)$ - obtained by using the time difference between the recent local maxima and recent local minima of the pitch rate.

Figure 3.26 defines how the recent minima and recent maxima were selected for the ROVER technique. For each of the four conditions, ROVER can assign a value of one or zero, based on whether certain thresholds corresponding to each of the four conditions are met. Then the ROVER integer value (RIV) is calculated by adding the values assigned by ROVER to each of the conditions. Therefore, the maximum value that RIV can have is four, which corresponds to severe HMO or instability. For proper use of the ROVER technique, it is important to properly select the threshold, which varies from aircraft to aircraft. This is one of the shortcomings of the ROVER technique, that the threshold values are dependent on task, aircraft, and configuration [38, 39]. The threshold values for the HuIL experiments were selected after considering all the pitch and joystick command data. Table 3.3 provides the threshold values used for the ROVER technique for the HuIL experiments, which were obtained after analyzing the data for 
TABLE 3.3: ROVER thresholds.

\begin{tabular}{cc}
\hline Conditions & Thresholds \\
\hline Magnitude of the pitch rate & $8 \mathrm{deg} / \mathrm{s}$ \\
Magnitude of the joystick deflection & $10 \mathrm{deg}$ \\
Phase difference & $65 \mathrm{deg}$ \\
Frequency of pitch rate & $1-8 \mathrm{rad} / \mathrm{s}$ \\
\hline
\end{tabular}
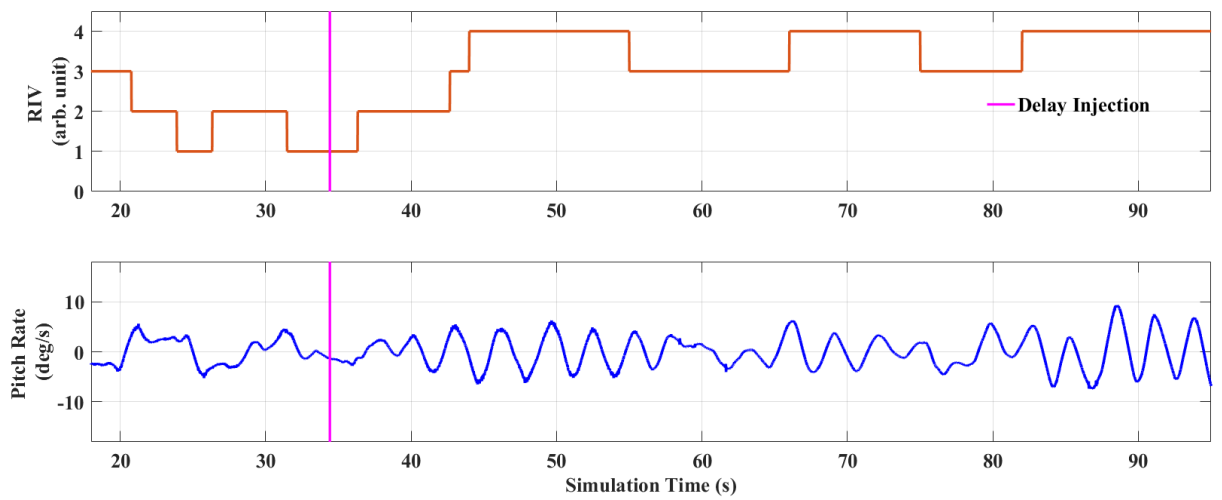

Figure 3.27: RIV for the HMS with time for HuIL experiment with 200ms time delay injection (Figure 3.21).

maximizing HMO detectability. Figure 3.27 presents the RIV values calculated using ROVER for the data shown in Figure 3.21.

It is evident from Figure 3.27 that, for the time interval in which Figure 3.25 predicts instability (i.e., stability metric values lie in the red region), the RIV value was predominantly four; hence, the ROVER technique detected strong oscillations. In addition, there was a clear delay in the ROVER technique in detecting the start of the oscillations. In summary, the significant overlap between the time interval in which the RIV value was four and the time interval in which Figure 3.25 predicted HMO provides validity to the RPStab results.

Another technique to detect real-time oscillations is wDFT, which is represented by the following equation [37]:

$$
\Delta X(\omega)_{t=T}=X(\omega)_{t=T}-X(\omega)_{t=T-k}
$$

In Eqn. 3.7, $X(\omega)_{t=T}$ is the discrete Fourier transform of the signal at time $t=T$, 


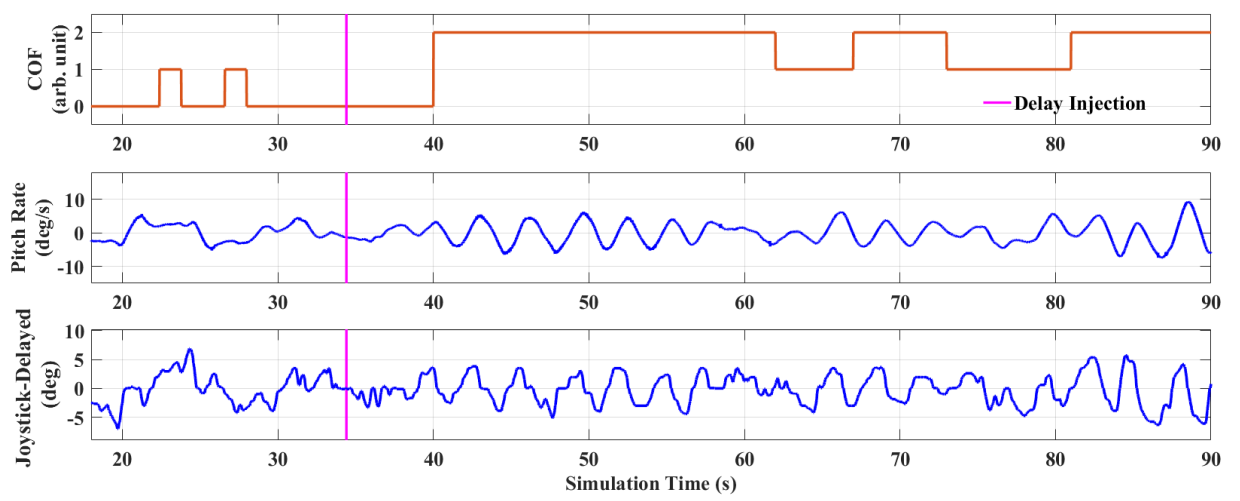

Figure 3.28: COF for HMS with time for HuIL experiment with $200 \mathrm{~ms}$ time delay injection (Figure 3.21).

and $X(\omega)_{t=T-k}$ is the discrete Fourier transform of the signal at time, $t=T-k$. The value of $\Delta X(\omega)_{t=T}$ provides a measure of the frequency content in the signal in the time window between $T$ and $T-k$. For oscillation detection, the norm of $\Delta X(\omega)_{t=T}$ was compared against a user-defined threshold to set an oscillation flag to indicate the presence of undesirable oscillations. Since the forcing function for the HuIL experiment was a sum of sines, the wDFT technique ensured that the forcing function frequencies were removed by means of subtraction, thereby preventing false oscillation flags due to the input forcing function. For this research, the wDFT technique was used to detect the presence of undesirable oscillations in both the pitch rate data and joystick command data because, in the HMO scenario, both the pitch rate data and joystick command would exhibit undesirable oscillatory tendencies. Similar to the ROVER technique, the wDFT technique assigns a value of one if it detects oscillations, and otherwise assigns a value of zero. Therefore, summing up the oscillations flag value from the pitch rate data and joystick command data, the maximum possible value for the combined oscillation flag (COF) is two. For this research, a time window of $3 \mathrm{~s}$ and threshold of $22.4 \mathrm{deg}$ were used after analysis of the experimental data and evaluating feedback from the subjects regarding the presence of oscillations. Figure 3.28 shows the detection results of the wDFT technique when applied to the data shown in Figure 3.21.

Similar to the ROVER detection results, there was a significant overlap between the regions in which RPStab predicted instability and the regions where the wDFT technique detected strong undesirable oscillation (i.e., COF value of two). It is interesting to note 
that, after the injection of the time delay, it took the wDFT technique some time to detect the presence of undesirable oscillations. This effect was due to a combination of the window length and the threshold used for the wDFT technique. A smaller window size would be able to capture more rapid changes in the system output, but would also increase the number of false flags. In summary, wDFT method results also support the predictions of RPStab method.

To evaluate the RPStab prediction results, the percentage value for each of the following conditions from the experimental data was calculated:

1. True positive (TP): system is predicted to show HMO, but the subject does not detect any $\mathrm{HMO}$

2. False negative (FN): system is predicted to not show HMO, but the subject detects $\mathrm{HMO}$

3. False positive (FP): system is predicted to not show HMO, but the subject detects $\mathrm{HMO}$

4. True negative (TN): system is predicted to show HMO and the subject detects HMO.

Table 3.4 provides the percentage value of the total time steps $(8,000$ sample points) for which the above four conditions occurred in the prediction results of the RPStab method for the HuIL experiment described in this section. Only HMO events (classified as such individually by three person familiar with the research) were considered true HMO events. Similar calculations were also performed for the ROVER and wDFT detection techniques. While calculating the values for RPStab, only the HCS with stability metric values in the red region were considered to show HMO. From the values in Table 3.4, the following values could then be calculated:

1. Total accuracy percentage (i.e., how often predictions/detections were correct): $\mathrm{TP}+\mathrm{TN}$.

2. TN percentage or HMO prediction accuracy ( i.e., how often predictions/detection of $\mathrm{HMO}$ were correct): $\mathrm{TN} /(\mathrm{TN}+\mathrm{FP})$. 
TABLE 3.4: Comparison of the RPStab results with ROVER and DFT detection results for the HuIL experiment data with time delay presented in this section.

\begin{tabular}{ccccc}
\hline Method & $\begin{array}{c}\text { True } \\
\text { Positive(\%) }\end{array}$ & $\begin{array}{c}\text { False } \\
\text { Negative(\%) }\end{array}$ & $\begin{array}{c}\text { False } \\
\text { Positive(\%) }\end{array}$ & $\begin{array}{c}\text { True } \\
\text { Negative(\%) }\end{array}$ \\
\hline RPStab & 27.17 & 10.33 & 5.83 & 56.67 \\
\hline ROVER & 27.19 & 9.56 & 8.14 & 55.11 \\
\hline wDFT & 26.41 & 10.67 & 7.85 & 55.07 \\
\hline
\end{tabular}

Table 3.5 provides the total accuracy, as well as the average accuracy and average TN percentage calculated for all the subjects for the HuIL experiments with time delay.

TABLE 3.5: Comparison of total accuracy and HMO prediction/detection accuracy.

\begin{tabular}{cccc}
\hline & Method & From Table 3.4 & $\begin{array}{c}\text { Average for all } \\
\text { subjects }\end{array}$ \\
\hline \multirow{2}{*}{$\begin{array}{c}\text { Total accuracy } \\
\text { (\%) }\end{array}$} & RPStab & 83.84 & 81.67 \\
& ROVER & 82.30 & 80.16 \\
\hline HMO predic- & RPStab & 81.48 & 80.24 \\
tion/detection & ROVER & 87.13 & 89.27 \\
accuracy (\%) & wDFT & 87.52 & 87.08 \\
\hline
\end{tabular}

The HMO prediction accuracy for the RPStab method was found to be similar to the HMO detection accuracy for the ROVER and wDFT techniques. However, the strength of the RPStab method lies in its ability to calculate margins and predict the stability characteristics of the HCS, instead of just detecting a HMO. Unlike the ROVER and wDFT techniques, for which thresholds need to be tuned for each subject and each configuration individually, once UKF was tuned initially, no further modifications were required for different subjects or experiments. This makes RPStab based on the UKF method more general and robust to variations, compared to detection techniques such as ROVER and wDFT. 


\subsection{Estimation Results for HuIL Experiments with Rate Limiting}

Similar to the time delay case, the UKF estimator was used to estimate the McRuer model parameters for the HuIL experiments undertaken with rate limiting on the joystick commands. For estimation, the pitch angle error and actual joystick output (i.e., joystick command before rate limiting) were used because the actual joystick output shows the true characteristics of human control behavior. This is expected as due to rate limiting the subject will change the joystick output to adapt to the changed system dynamics. Since rate limiting is a nonlinear phenomenon, linear control theory analysis tools cannot be used to predict instability. Instead, describing function analysis was performed to identify limit cycles and compare them with the actual experimental data. Figure 3.29 shows a set of pitch angle and joystick command values for the HuIL experiment with rate limiting used in the estimation process to show the subject control behavior.
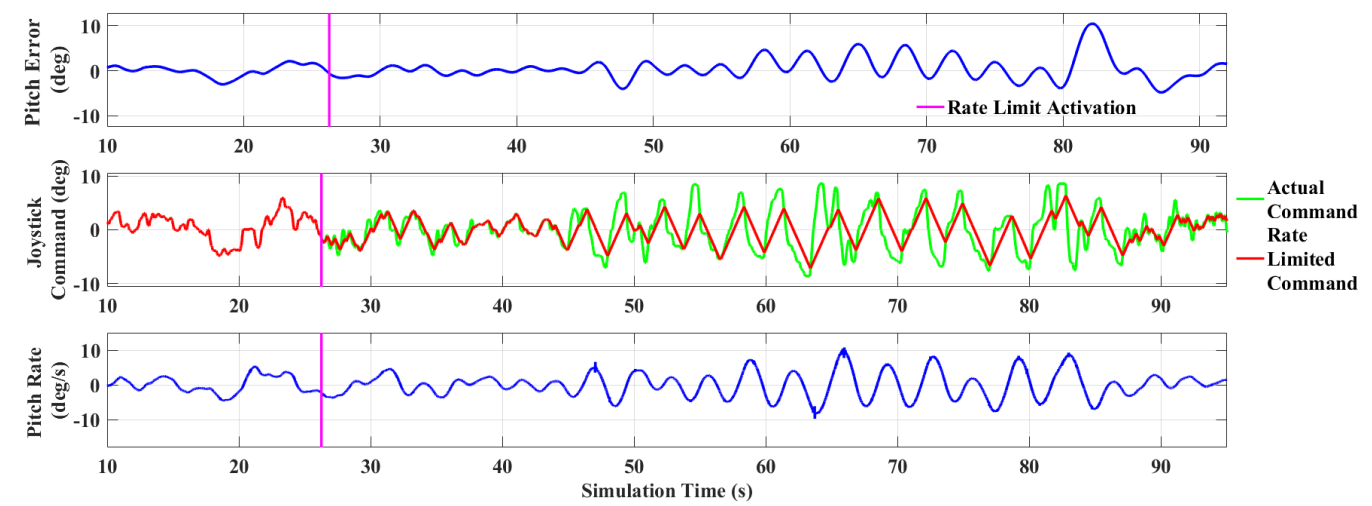

FIGURE 3.29: Pitch, joystick command, and pitch rate data for HuIL experiment with $10 \mathrm{deg} / \mathrm{s}$ rate limiting.

It was observed that the subject had difficulty performing the task immediately after the rate limiting was activated (due to the subject moving the joystick faster than the maximum rate permitted). The standard deviation of the pitch error before activation of the rate limiting for the data shown in Figure 3.29 was found to be $0.83 \mathrm{deg}$, and the standard deviation of the pitch error post-activation of the rate limiting was found to be $4.09 \mathrm{deg}$. The standard deviation of the joystick commands for the two section were found to be 1.38 , and $4.79 \mathrm{deg}$, respectively which further provides information about 

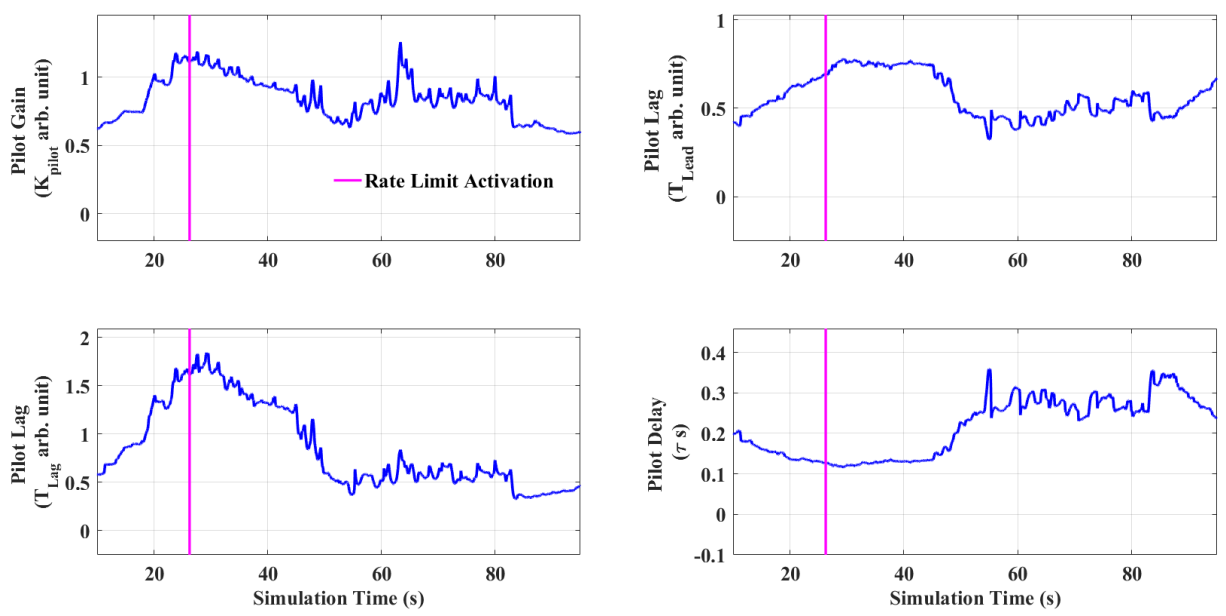

FiguRE 3.30: Estimation results for data shown in Figure 3.29.

the increase in task difficulty along with strong oscillatory tendencies seen in Figure 3.29 post rate limiting.

Rate limiting is nonlinear in nature and frequency analysis techniques cannot be used after the activation of the rate limiting to analyze the stability of the HCS. However, frequency analysis for the time interval before the rate limit activation was performed to attain the initial system stability characteristics. As before, the McRuer model was obtained using the parameters calculated by the UKF algorithm in real-time. Figure 3.30 shows the McRuer model parameters obtained for the data shown in Figure 3.29.

It can be observed that the delay $(\tau)$ increased and the lag $\left(T_{L a g}\right)$ decreased after the activation of the rate limiting. The decrease in the lag can be thought of as the subject countering the increase in lag due to rate limiting. Figures 3.31 and 3.32 show the variation in margins and crossover frequencies with time until the activation of rate limiting to obtain information regarding the stability characteristics of the HCS, which is expressed as the stability metric value plot in Figure 3.33. From Figure 3.33, the HCS was predicted to be stable and not show any oscillation tendencies before the activation of the rate limiting, and the HCS was found to have sufficient gain and phase margins.

Describing function technique was used to analyze the stability of the HCS and the existence of the limit cycles after the activation of rate limiting. A detailed description of the describing function techniques can be found in [40, 41, 42]. The describing function 

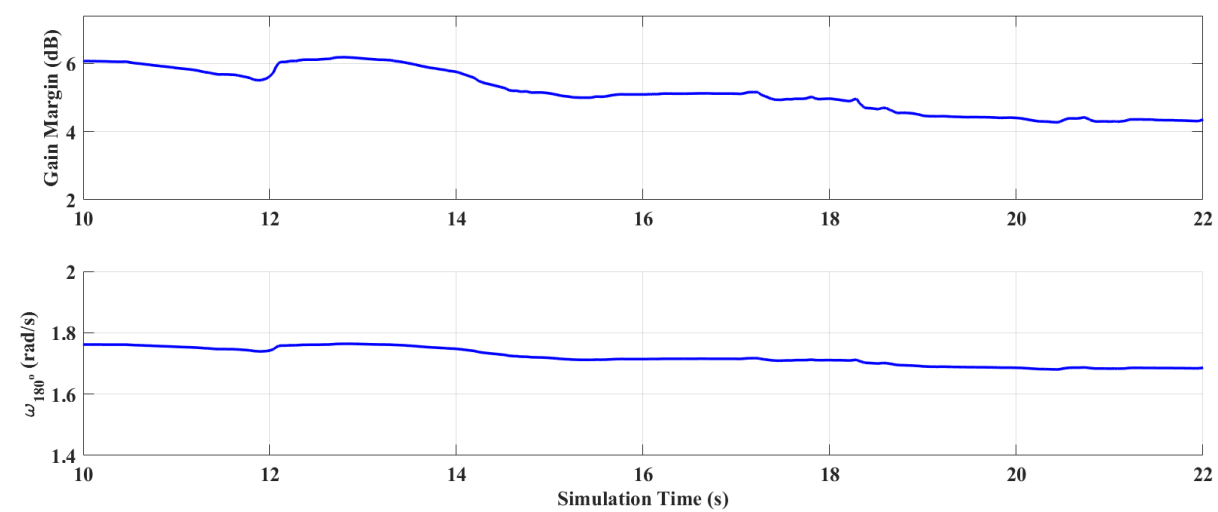

FIGURE 3.31: Change in gain margin and phase crossover frequency with time for the experiment represented in Figure 3.29 before the activation of rate limiting.
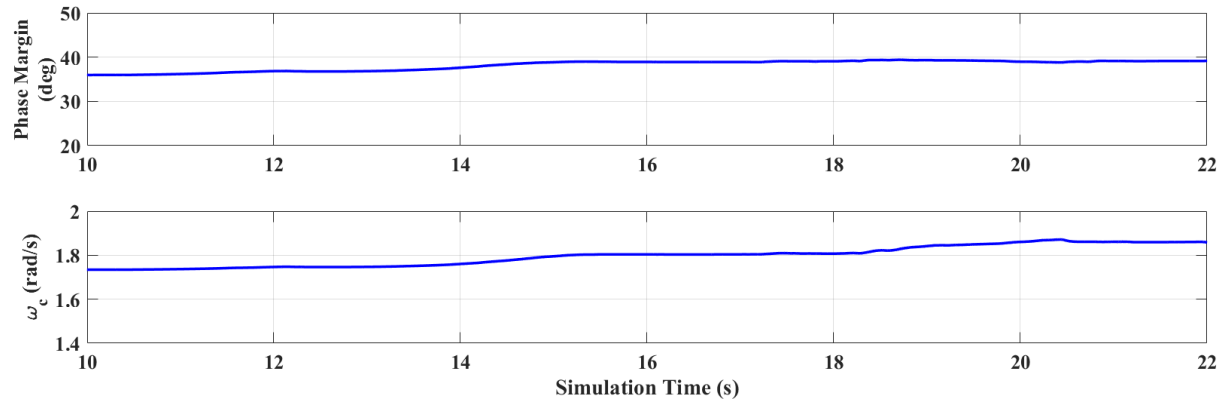

FIGURE 3.32: Change in phase margin and gain crossover frequency with time for experiment represented in Figure 3.29 before activation of rate limiting.
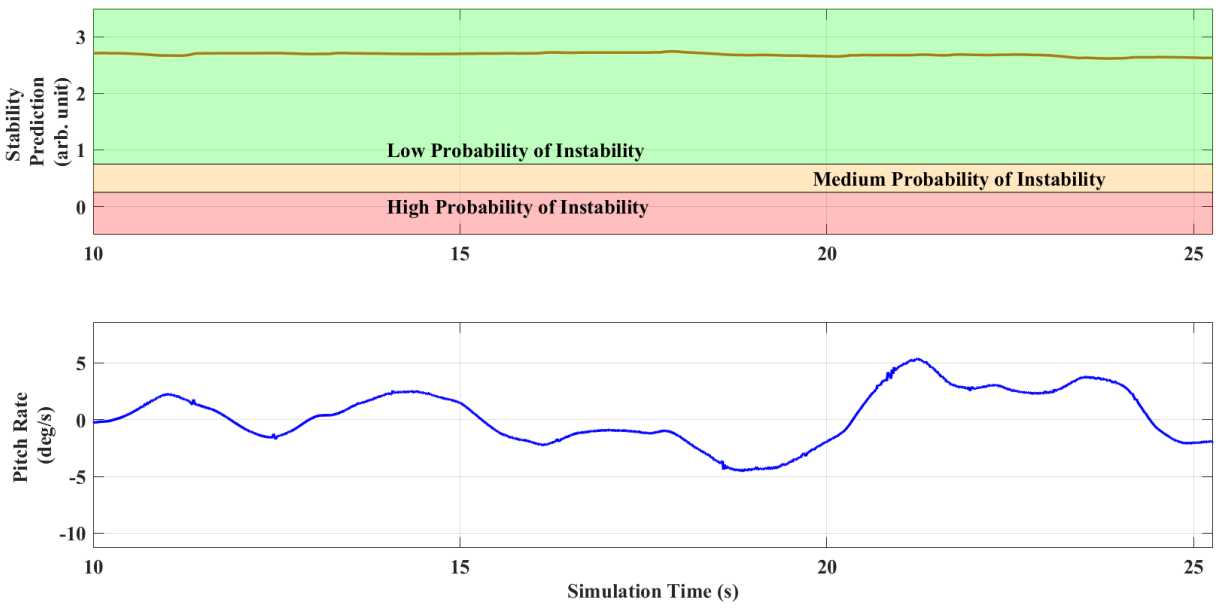

FiguRE 3.33: Stability value of the closed-loop HCS with time for HuIL experiment before the activation of rate limiting. 


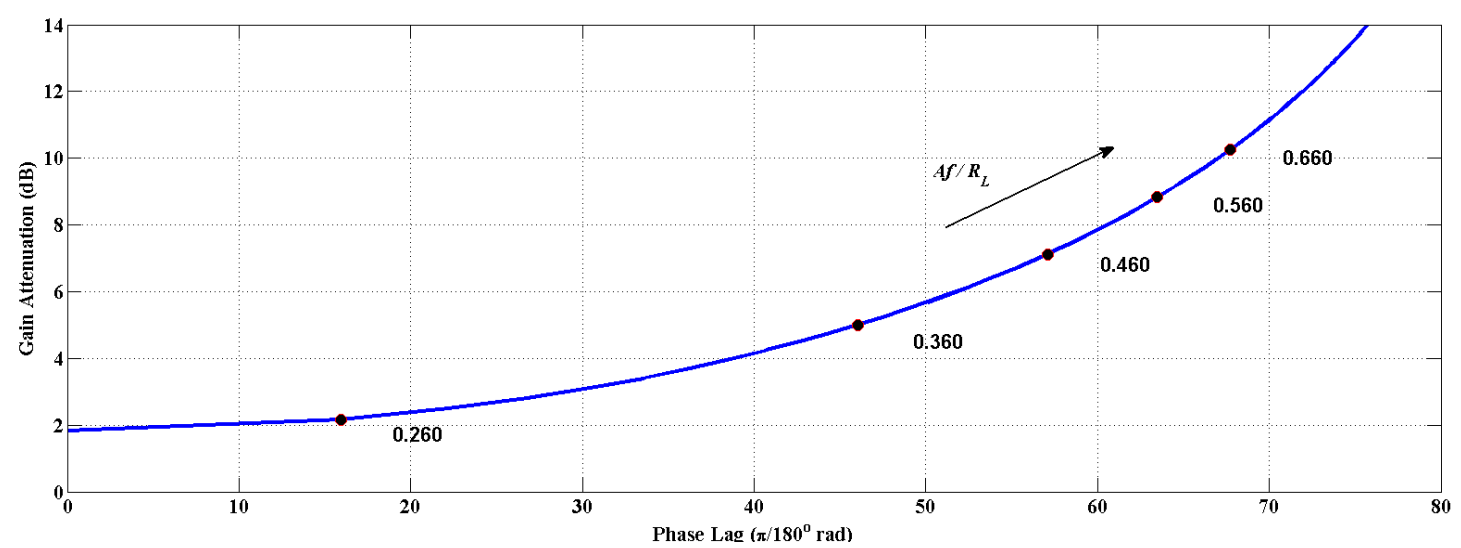

Figure 3.34: Rate limit describing function characteristics.

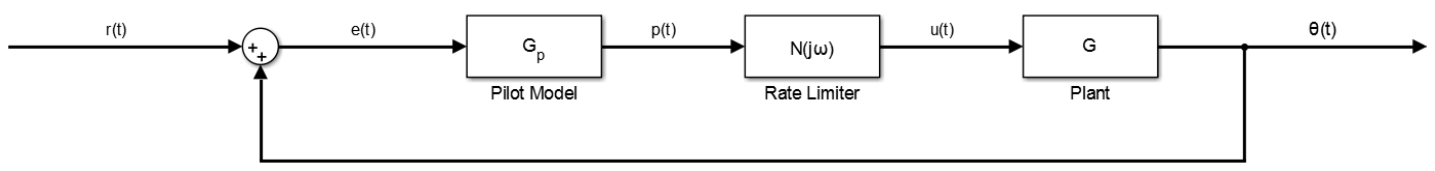

FiguRE 3.35: Closed loop HCS with rate limiting.

equations for rate limiting are given by the following:

Phase lag due to rate limiting: $\angle(\omega)=\phi=\sin ^{-1} \frac{R_{L}}{4 A f}-\frac{\pi}{2}$

Gain attenuation due to rate limiting: $|N(\omega)|=\frac{2 R_{L}}{\pi^{2} A f}=\frac{8}{\pi^{2}} \sin \left(\phi+\frac{\pi}{2}\right)$

Where $R_{L}$ is the maximum rate permitted, $A$ is the amplitude of the input, and $f$ is the frequency of the input. As the rate limit value $\left(R_{L}\right)$ decreased, the gain approached zero and the phase lag approached 90 degrees. Since both gain and phase of the describing function are dependent on the ratio $\frac{R_{L}}{A f}$, it is easier to plot the describing function phase lag and gain attenuation in $\mathrm{dB}$ as $\frac{A f}{R_{L}}$ varies, as shown in Figure 3.34.

Figure 3.35 shows the closed loop system with rate limiting used for the limit cycle analysis along with the describing function. The closed loop system shown in Figure 3.35 is described by;

$$
\frac{G_{p}(j \omega) N(j \omega) G(j \omega)}{1+G_{p}(j \omega) N(j \omega) G(j \omega)}
$$

In the above equation $G_{p}(j \omega)$ is the estimated human control model and $G(j \omega)$ is the plant transform function. For sustained oscillations, the following equation should be 


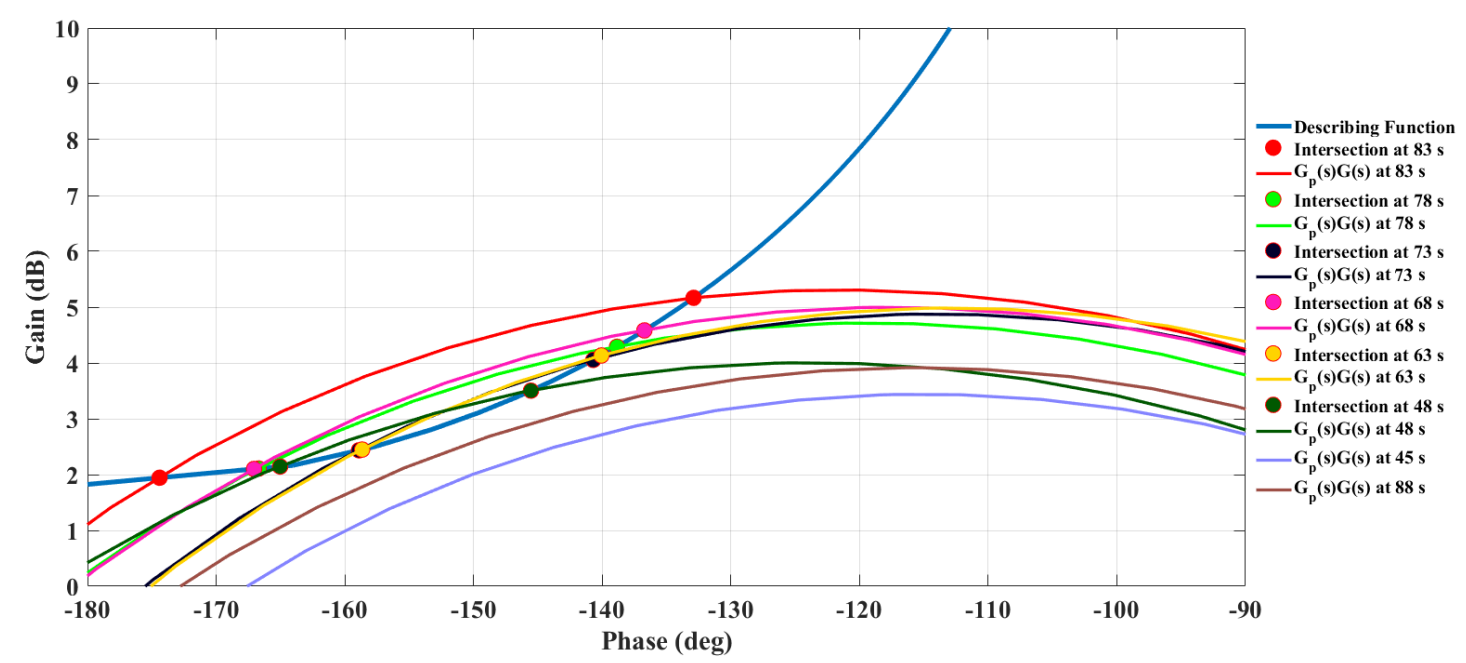

FIGURE 3.36: Nichols plot of the open loop system and inverse describing function at different time intervals.

satisfied:

$$
\begin{aligned}
1+G_{p}(j \omega) N(j \omega) G(j \omega) & =0 \\
G_{p}(j \omega) G(j \omega) & =\frac{-1}{N(j \omega)}
\end{aligned}
$$

Thus, the solution for Eqn. 3.10 is the intersection points between the Nichols plot of $G_{p}(j \omega) G(j \omega)$ and the Nichols plot of the negative reciprocal of the describing function. The intersection points provide the frequency of the limit cycles, corresponding gain attenuation, and phase lag values. For the experiment represented in Figure 3.29, $G_{p}(j \omega) G(j \omega)$ intersects the $\frac{-1}{N(j \omega)}$ at $48 \mathrm{~s}$ for the first time, which is $\sim 20 \mathrm{~s}$ after the activation of the rate limiting. Figure 3.36 shows the $G_{p}(j \omega) G(j \omega)$ and $\frac{-1}{N(j \omega)}$ Nichols plot at discrete points in the interval 28-90 s. Figure 3.36 also shows the intersection points if there are any. The open loop system $G_{p}(j \omega) G(j \omega)$ intersects with $\frac{-1}{N(j \omega)}$ in the time interval 48-83 s and, looking at Figure 3.29, the HCS does show strong oscillatory tendencies during this time interval. It needs to be verified that the limit cycle frequency predicted by the describing function analysis matches the actual frequency of the oscillations. It is also interesting to note that the describing function analysis predicts two possible limit cycles for each time that the two curves intersect. Table 3.6 presents the limit cycle frequencies predicted in different intervals, where $\omega_{L C_{1}}$ and $\omega_{L C_{2}}$ are the two limit cycle frequencies predicted.

There was no significant difference between $\omega_{L C_{1}}$ and $\omega_{L C_{2}}$. In addition, both the 
TABLE 3.6: Limit cycle frequency at different time from Figure 3.36.

\begin{tabular}{ccccc}
\hline Time (Sec) & $\omega_{\mathbf{L C}_{\mathbf{1}}}(\mathbf{r a d} / \mathbf{s})$ & $\frac{\mathbf{A f}}{\mathbf{R}_{\mathbf{L}}}$ & $\omega_{\mathbf{L C}_{\mathbf{2}}}(\mathbf{r a d} / \mathbf{s})$ & $\frac{\mathbf{A f}}{\mathbf{R}_{\mathbf{L}}}$ \\
\hline 45 & $\mathrm{~N} / \mathrm{A}$ & $\mathrm{N} / \mathrm{A}$ & $\mathrm{N} / \mathrm{A}$ & $\mathrm{N} / \mathrm{A}$ \\
48 & 1.72 & 0.23 & 1.45 & 0.32 \\
63 & 1.68 & 0.22 & 1.48 & 0.30 \\
68 & 1.70 & 0.22 & 1.44 & 0.32 \\
73 & 1.67 & 0.21 & 1.48 & 0.30 \\
78 & 1.71 & 0.23 & 1.45 & 0.32 \\
83 & 1.76 & 0.25 & 1.41 & 0.35 \\
88 & $\mathrm{~N} / \mathrm{A}$ & $\mathrm{N} / \mathrm{A}$ & $\mathrm{N} / \mathrm{A}$ & $\mathrm{N} / \mathrm{A}$ \\
Average & 1.71 & 0.23 & 1.45 & 0.32 \\
\hline
\end{tabular}

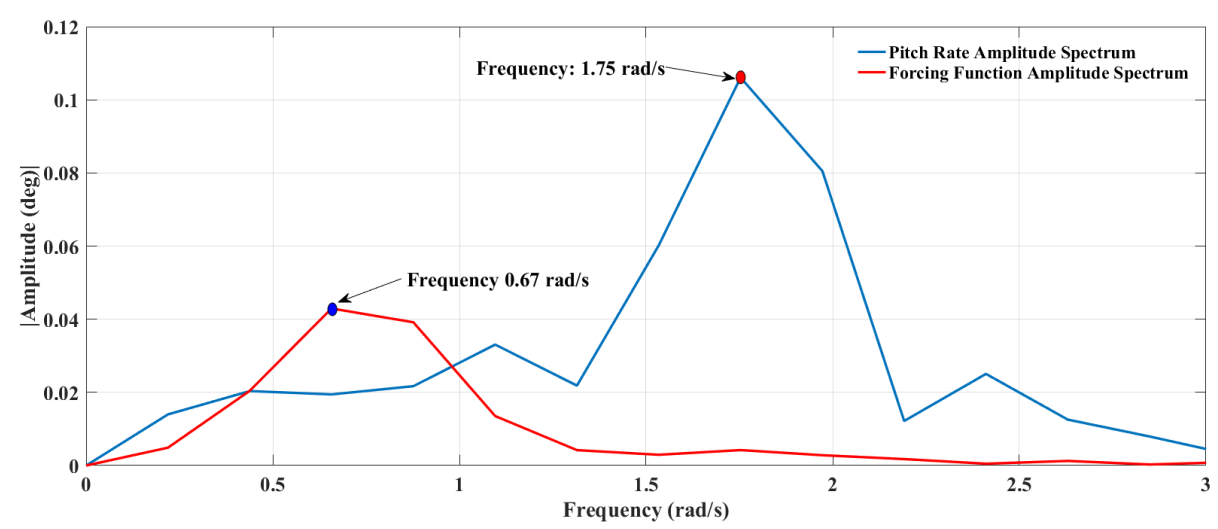

Figure 3.37: Amplitude spectrum of pitch rate and forcing function for time interval 25-40 s for the data in Figure 3.29

limit cycle frequencies were in the vicinity of the typical crossover frequency of the HCS observed during the HuIL experiments under nominal conditions. The calculated limit cycle frequencies were compared with the actual frequency of the oscillations in the data to validate the prediction. Figure 3.37 shows the amplitude spectrum of the pitch rate and input forcing function for the time interval of 48 to $83 \mathrm{~s}$. the amplitude spectrum of the pitch rate for the time interval of $48-83 \mathrm{~s}$ had a peak at $1.75 \mathrm{rad} / \mathrm{s}(0.28 \mathrm{~Hz})$, which was close to the average $\omega_{L C_{1}}$ value of $1.71 \mathrm{rad} / \mathrm{s}(0.27 \mathrm{~Hz})$ obtained from Table 3.6, as predicted by using the describing function analysis. In addition, the amplitude spectrum of the forcing function is shown in Figure 3.37 to indicate that the oscillations in pitch rate are not an artifact of the sum of sines input. For the average $\omega_{L C_{2}}$ value of $1.49 \mathrm{rad} / \mathrm{s}(0.24 \mathrm{~Hz})$, the value of $A$ that would lead to oscillations was calculated to 

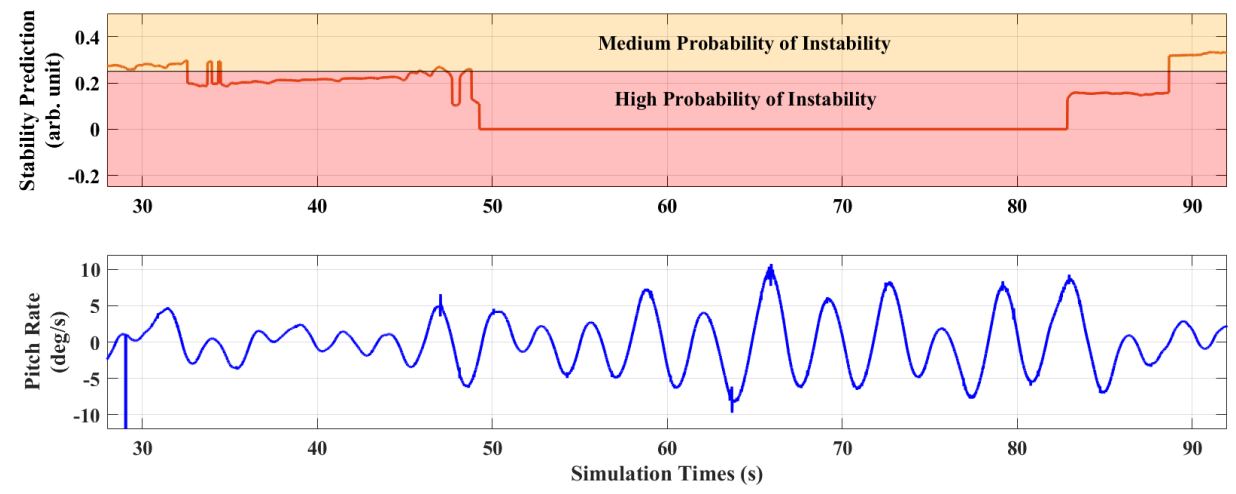

FIGURE 3.38: Stability value of closed-loop HCS with time (after the activation of rate limiting) for HuIL experiment with $10 \mathrm{deg} / \mathrm{s}$ rate limiting.

be approximately 11.85 deg. Due to the way forcing function has been designed, it can never reach the value of $11.25 \mathrm{deg}$, which shows HCS oscillates at a frequency closer to $\omega_{L C_{1}}$

For describing function evaluation the HuIL experiments with rate limiting HCS were also assigned a stability metric value for each time step, based on the following equation:

$$
S_{k}= \begin{cases}\frac{\Delta K}{K_{\text {pilot }}}, & \text { if limit cycle not predicted } \\ 0, & \text { if limit cycle predicted and is feasible }\end{cases}
$$

In Eqn. 3.11, $S_{k}$ is the stability metric value of the HCS at time step $k$, and $\Delta K$ is the increment in the gain needed to intersect Nichols plot of $G_{p}(j \omega) G(j \omega)$ with the Nichols plot of the negative reciprocal of the describing function. $\Delta K$ provides information about how close the system is from having a limit cycle. A higher value of $\Delta K$ means that the gain must be increased by large amounts to result in limit cycles; thus, a HCS with high $\Delta K$ is less likely to oscillate, and vice-versa. Figure 3.38 shows the change in stability values of the closed loop HCS with time, after the activation of rate limiting.

Like earlier HuIL experiments, instability regions were obtained by analyzing the experimental data for the stability metric values obtained using Eqn. 3.11. It can be seen that (Figure 3.38), after the activation of rate limiting, the stability value moved into the red region and the pitch rate data showed the start of oscillations. For the time interval (48 - 83 s) in which the HCS was predicted to have limit cycles, the HCS showed strong oscillatory behavior. Around $88 \mathrm{~s}$, the oscillation died out and the stability value moved 



FIGURE 3.39: RIV for HCS with time for HuIL experiment after activation of $10 \mathrm{deg} / \mathrm{s}$ rate limit (Figure 3.29).
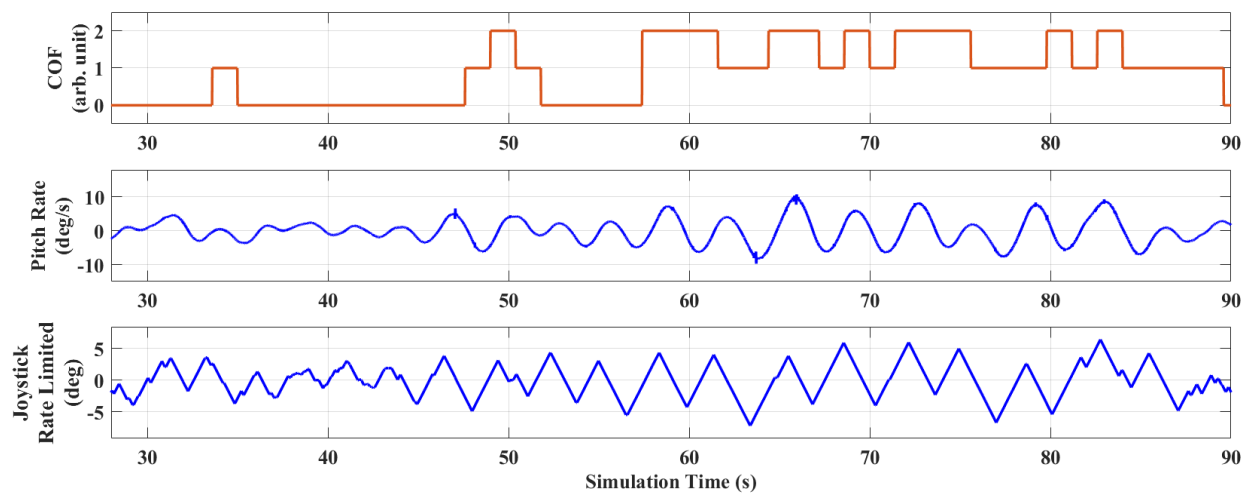

Figure 3.40: COF for HCS with time for HuIL experiment after activation of $10 \mathrm{deg} / \mathrm{s}$ rate limit (Figure 3.29).

into the orange region. The stability value after $88 \mathrm{~s}$ kept increasing and the pitch rate data also showed no oscillations after $88 \mathrm{~s}$. Therefore, it can be said that the describing function technique was able to capture the stability characteristics of the HCS.

Similar to the HuIL experiments with time delay, the ROVER and wDFT techniques were used to detect HMO in the pitch rate data and were compared with the realtime prediction of limit cycle (RPLC) from the estimated McRuer model parameters. Figure 3.39 shows the ROVER analysis after the activation of the rate limiting for the data shown in Figure 3.29. It can be seen that there was strong agreement between the ROVER detection and RPLC results. To further support the prediction result of the RPLC method, oscillation detection was performed using the wDFT technique Figure 3.40. The wDFT technique also supported the predictions made by the RPLC method and detection by the ROVER technique. Since both the ROVER and wDFT technique detection results supported the prediction made by the RPLC method using the estimated McRuer model, it can be stated that the parameters estimated by UKF 
TABLE 3.7: Comparison of the RPLC method results with ROVER and DFT detection results for HuIL experiment with rate limiting presented in this section.

\begin{tabular}{ccccc}
\hline Method & TP (\%) & FN (\%) & FP (\%) & TN (\%) \\
\hline RPLC & 17.33 & 10.32 & 2.11 & 70.24 \\
ROVER & 19.74 & 7.30 & 3.25 & 69.71 \\
wDFT & 20.13 & 7.67 & 3.28 & 68.92 \\
\hline
\end{tabular}

TABLE 3.8: Comparison of total accuracy and HMO prediction/detection accuracy for $\mathrm{HuIL}$ experiments with rate limiting

\begin{tabular}{cccc}
\hline & Method & For data in Table 3.7 & $\begin{array}{c}\text { Average for all } \\
\text { subjects }\end{array}$ \\
\hline Total accuracy & RPLC & 87.57 & 86.79 \\
(\%) & ROVER & 89.45 & 87.61 \\
& wDFT & 89.05 & 87.03 \\
\hline HMO predic- & RPLC & 97.08 & 95.11 \\
tion/detection & ROVER & 95.56 & 94.51 \\
accuracy (\%) & wDFT & 95.46 & 94.91 \\
\hline
\end{tabular}

predicted the stability characteristics of the HCS with sufficient accuracy, and predicted the frequency of the oscillation of the limit cycles.

To assess the prediction performance for the rate limiting case, Table 3.7 provides the percentage value of the total sample points for which each conditions (TP, FN, FP, and TN) occurred post-activation of rate limiting for the data shown in Figure 3.29. Table 3.8 provides the total accuracy and TN percentage for the data provided in Table 3.7, and provides the average accuracy and average TN percentage calculated for 11 subjects for HuIL experiments with rate limiting.

The RPLC accuracy is similar to the other two methods. An argument similar to that described in Section 3.6 also holds here. The added benefit of using the RPLC method was its prediction of possible limit cycle frequencies (i.e., the frequency of HMO), even without considering the full window of oscillations. In addition, the ROVER and wDFT techniques did not incorporate any information about the rate limiting nonlinearity present in the system as compared to the RPLC method. 


\subsection{Prediction vs. Detection}

The goal of this short section is to properly distinguish between HMO prediction and detection techniques to emphasize the contribution of RPStab and RPLC methods. The detection values obtained using the ROVER and wDFT techniques were based on the analysis of past data. In contrast, RPStab and RPLC methods incorporate information about the underlying model obtained from UKF algorithm. This underlying model enables prediction of future events given an input. In addition, the stability metric value provided by RPStab and RPLC are continuous, which can provide information regarding future HMO events (e.g., the onset of HMO events) by its location and movement trend in the criteria plane - see Table 3.2. However, the current work did not implement this prediction technique, rather it is shown that the UKF algorithm was able to determine HCS stability characteristics.

\section{7 $\quad$ Statistical Anaysis}

Post-hoc power analysis was undertaken on HMO Prediction Accuracy Percentage (PA\%) to obtain the statistical power of the results from previous sections with 11 subjects. The results from 15 trials were averaged for each individual subject, and then a one-sample t-test was performed.

This kind of analysis comes at the cost of loss of information due to averaging of data. However, it provides a straightforward way of conducting a t-test, as the overall mean of HMO PA\% for the population (i.e., all human controllers) from which the subjects were sampled was of primary importance as opposed to the variation in HMO PA\% with trials for a single individual. The aggregate mean of HMO PA\% was found to be $86.28 \pm 7.57 \%$.

Typical ROVER accuracy was reported to be between $75-85 \%$ in existing literature, and it is desired that the UKF algorithm accuracy is similar in predicting HMO. Evidence from the right tail one-sample t-test for a null mean of $75 \%$ for HMO PA\% showed a lack of statistical power $\left({ }^{*} \mathrm{p}>0.05\right)$. Therefore, the results carry only observational subjective 
weight, and the statistical power can be improved in future studies with a larger number of participants.

\subsection{Summary}

This chapter provided a description of the HuIL experiments' setup to validate the prediction capability of the UKF estimator designed in Chapter 2. The HuIL experiments enabled the researchers to have better control over the experimental parameters and properly constrain the problem to accurately determine the effectiveness of the UKF estimator. The HuIL experiments were performed for three different cases:

1. Experiments with only nominal plant and subjects.

2. Experiments with delay injection between subjects and the plant.

3. Experiments with rate limiting of subjects' joystick command to the plant.

The plant used was a short period longitudinal mode of an aircraft, typical of a small business jet. No HMOs were seen for the experiments without delay or rate limiting, and the McRuer model obtained using UKF-estimated parameters was able to correctly predict the system stability for all these cases.

For the HuIL experiments with injection of time delay, the RPStab HMO prediction results were similar to the HMO detection results from the ROVER and wDFT techniques. However, the added benefit of RPStab is its potential ability to predict stability and calculate the gain and phase margins to attain better knowledge of the stability of HCS.

For the HuIL experiments with rate limiting, describing function technique was used to express the rate-limiting non-linearity to be able to use frequency domain techniques, such as the Nichols plot, to undertake limit cycle analysis. The results indicated that the HCS oscillated at a value close to that predicted by the RPLC method using the estimated McRuer model parameters from the UKF algorithm. In addition, the limit cycle analysis method provided prediction results similar to the detection results from 
the ROVER and wDFT techniques. Therefore, this chapter helped establish the validity of the UKF estimator's capabilities and usefulness in predicting HCS stability for a single-input single-output manual compensatory task. 


\section{Chapter 4}

\section{Conclusions}

$\mathrm{T}$

his dissertation presented a novel technique to estimate McRuer model parameters in real-time. The primary motivation for the work presented in this dissertation was that the estimated pilot model can be used to determine closed-loop HCS stability in real-time. With further development this technique could be useful in predicting the onset of LOC events and could notify the pilot to take preemptive actions to prevent such events. This study focused on a simplified problem, that is SISO manual control. For a SISO reactive compensatory task (i.e. with a goal of error reduction), the human controller is approximated with a McRuer model comprising of a gain, a lead parameter, a lag parameter, and a time delay. Therefore, the estimation framework becomes a problem of identifying these parameters in real-time. Previous efforts to use the McRuer model to study human controllers have focused primarily on off-line batch estimation of the model parameters from the experimental data. This essentially assumes the human controller to be time-invariant during the course of the experiment; however, this is not always the case and batch estimation methods fails to incorporate the adaptive nature of humans. Instead, this dissertation presented an algorithm using UKF to estimate the McRuer model parameters in real-time. To evaluate the estimator capabilities, simulation studies were performed which showed that the estimator was able to estimate actual parameters with sufficient accuracy. It was also found that the estimator preserves the closed-loop HCS stability characteristics under linear modeling errors, such as when the 
human controller does not behave exactly as stated in the McRuer model. The estimator was further validated using actual human control data from HuIL experiments.

In HuIL experiments, 11 human subjects were given the task of error (displayed on a screen) reduction using a joystick. This ensured that the researcher had proper control of the experiments and could negate plant modeling errors. The experiments were performed for the time delay injection in the HCS and rate limiting of the joystick command. The experimental data were fed into the UKF estimator to obtain the McRuer model parameters to make predictions about the closed-loop HCS stability in real-time. It was observed that this technique provided sufficiently accurate ( $\sim 85 \% \mathrm{PA})$ assessment regarding the presence and onset of instabilities in the HCS. This technique was further compared with the ROVER and wDFT techniques for detecting oscillations. The comparison showed that the RPStab result agreed with the results from ROVER and $\mathrm{wDFT}$.

To summarize, the major contributions of this dissertation were as follows:

1. Estimation of human control model parameters using UKF in real-time

2. Prediction of instability in a closed-loop human-machine system for pitch control using HuIL experiments.

\subsection{Limitations and Future Work}

This work has demonstrated the possibility of predicting the stability of closedloop HCS in real-time. However, this research direction requires further study, as the work in this dissertation was limited to single-input single-output reactive compensatory tracking problems. It is necessary to employ multiple-input single-output estimators to satisfactorily model human pilots in real flight. It is also challenging to design a flight test experiment while keeping control of all the conditions such as environment, plant modeling errors etc. Higher fidelity HuIL experiments can be used as pre-flight tool to better design the flight experiments. In addition, the work in this dissertation has indicated that the UKF estimator provides accurate stability information under linear 
modeling errors, and that further analysis needs to be undertaken for nonlinear modeling errors, such as dead band in human control behavior for a more complete prediction method. In future work, to detect onset of LOC events the prediction method discussed in Section 3.6 should be implemented. In addition, more human subjects need to be recruited for the HuIL experiments to improve the statistical power of the result. All this will ensure that when the estimator is applied to real world problems it will provide accurate results and help in improving the safety of systems such as cars, airplanes etc. 


\section{Appendix A}

\section{IRB Procedure and Approval}

he following procedure was submitted to IRB to obtain approval for HuIL experiments.

1. Recruiting: potential participants will be contacted verbally in the engineering building and asked if they are interested in participating (see attached recruitment script). If they are interested, they will be asked to come to the lab.

2. Consent: the experiment procedure will be described in detail and if the potential participants are still interested we will ask them to sign a consent form. A date and time for participation will then be arranged.

3. Collecting data: the experiment apparatus consists of a joystick and a normal desktop computer. During the experiment human participants (one at a time) will sit in front of a computer screen which will display the position of a ball along with the position of a circle. Human participants will be asked to use a video game joystick to control the position of the ball and try to maintain the ball's position within the circle. The procedure is like playing a video game and this is a minimal risk experiment. Only the joystick movement will be saved on the computer. Each experiment run will be 2 min long. Between 10 to 15 runs will be performed in each session, which will take a total about $32-45$ minutes. It is not possible to trace participant's personal information from the results. There are no surveys or 
questionnaires and no videos will be recorded, participants will be only asked to move the joystick.

4. How findings will be shared: the findings of the aggregate result regarding the effectiveness of the method developed in this research will be published in conference and journal publications. However, no personal information about the human participants will be shared at any time.

\section{W. WestVirginiaUniversity. \\ Office of Research Integrity and Compliance \\ 886 CHESNUT RIDGE ROAD MORGANTOWN, WV 26506 \\ Approval Letter Expedited}

$\begin{array}{ll}\text { Action Date } & 10 / 13 / 2016 \\ \text { To } & \text { Yu Gu } \\ \text { From } & \text { WVU Office of Research Integrity and Compliance } \\ \text { Approval Date } & 10 / 13 / 2016 \\ \text { Expiration Date } & 10 / 12 / 2017 \\ \text { Subject } & \text { Protocol Approval Letter } \\ \text { Protocol Number } & 1609282859 \\ \text { Title } & \text { Data Collection Using a Joystick and a Computer }\end{array}$

The above-referenced research study was reviewed by the West Virginia University Institutional Review Board IRB and was approved in accordance with 46 CFR 46.101b.

It has been determined that this study is of minimal risk and meets the criteria as defined by the expedited categories listed below:

Documents reviewed and/or approved as part of this submission:

ConsetForm_wo_HIPAA_TanmayV1_Submit.pdf: 2016-10-07-04:00

Computer Display to be Used during the Experiment_Submit.pdf: 2016-10-07-04:00

RecruitmentScrip_Tanmay_V3_Submit.pdf: 2016-10-07-04:00

Documents for use in this study are available in the WVUkc system in the Notes and Attachments section of your protocol.

The Office of Research Integrity and Compliance is here to provide assistance to you from the initial submission of an IRB protocol and all subsequent activity. Please feel free to contact us by phone at 304.293.7073 with any question you may have. Thank you.

WVU Office of Research Integrity and Compliance 
Date:10/13/2016

Signed:



Lilo Ast

Senior Program Coordinator

Once you begin your human subject research, the following regulations apply:

1. Unanticipated or serious adverse events/side effects encountered in this research study must be reported to the IRB within five (5) days via the Notify IRB action.

2. Any modifications to the study protocol or informed consent form must be reviewed and approved by the IRB prior to implementation via submission of an amendment.

3. You may not use a modified informed consent form until it has been approved and validated by the IRB. 


\section{Bibliography}

[1] G. Padfield and B. Lawrence. "The Birth of Flight Control:An Engineering Analysis of the Wright Brothers' 1902 Glider". In: The Aeronautical Journal, Department of Engineering, The University of Liverpool Vol. 107.No. 1078 (Jan. 2008).

[2] D. McRuer et al. "Human pilot dynamics in compensatory systems. theory, models and experiments with controlled element and forcing function variations". In: Wright-Patterson AFB (OH): Air Force Flight Dynamics Laboratory (1965).

[3] D. McRuer and D. Weir. "Theory of manual vehicular control". In: Ergonomics 12.4 (1969), pp. 599-633.

[4] R. Hosman and H. Stassen. "Pilot's perception in the control of aircraft motions". In: Control engineering practice 7.11 (1999), pp. 1421-1428.

[5] D. McRuer. Pilot-Induced Oscillations and Human Dynamic Behavior. 4683. NASA, (1995).

[6] SAAB-SCANIA AB. SAAB Experience with PIO. 335. AGARD, (1995).

[7] MA. Dornheim. "Report pinpoints factors leading to YF-22 crash". In: Aviation Week and Space Technology Vol. 137.No. 19 (1992).

[8] Military Standard. Flying Qualities of Piloted Aircraft. MIL-STD-1797A. Department of Defense, (1990).

[9] MA. Dornheim. "Boeing Corrects Several 777 PIOs". In: Aviation Week and Space Technology Vol. 142.No. 19 (1995).

[10] W. Norton. Aeroelastic Pilot-In-The-Loop Oscillations. PIO Workshop following Active Control Technology: Applications and Lessons Learned,(Turin, Italy). AGARD, (1994). 
[11] R. Hoh, D. Mitchell, and J. Hodgkinson. "Bandwidth: A criterion for highly augmented airplanes". In: AGARD Criteria for Handling Qualities of Mil. Aircraft 11 p(SEE N 83-10054 01-08) (1982).

[12] R. Hess and R. Kalteis. "Technique for predicting longitudinal pilot-induced oscillations". In: Journal of Guidance, Control, and Dynamics 14.1 (1991), pp. 198204.

[13] F. Green and S. Hoffmann. "Design and development of a three-axis augmentation system for a Class III STOL assault transport". In: AIAA, AHS, and ASEE, Aircraft Systems, Design and Technology Meeting, Dayton, OH. (1986).

[14] M. Land and D. Lee. "Where do we look when we steer." In: Nature (1994).

[15] C. Sentouh et al. "A sensorimotor driver model for steering control". In: Systems, Man and Cybernetics, 2009. SMC 2009. IEEE International Conference on. IEEE. (2009), pp. 2462-2467.

[16] R. Hess and A. Modjtahedzadeh. "A control theoretic model of driver steering behavior". In: Control Systems Magazine, IEEE 10.5 (1990), pp. 3-8.

[17] D. McRuer and H. Jex. "A review of quasi-linear pilot models". In: Human Factors in Electronics, IEEE Transactions on 3 (1967), pp. 231-249.

[18] S. Shappell and D. Wiegmann. A human error approach to aviation accident analysis: The human factors analysis and classification system. Ashgate Publishing, Ltd., (2012).

[19] D. Kleinman, S. Baron, and W. Levison. "A control theoretic approach to mannedvehicle systems analysis". In: Automatic Control, IEEE Transactions on 16.6 (1971), pp. $824-832$.

[20] L. Taylor. "A Comparison of Human Response Modeling in the Time and Frequency Domains". In: Third Annual NASA-University Conference on Manual Control, NASA SP-144. (1967), pp. 137-153.

[21] N. Ninz. "Parametric identification of human operator models". In: NASA. Ames Research Center 16 th Ann. Conf. on Manual Control. (1982), pp. 137-145. 
[22] D. Pool et al. "Pilot equalization in manual control of aircraft dynamics". In: Systems, Man and Cybernetics, 2009. SMC 2009. IEEE International Conference on. IEEE. (2009), pp. 2480-2485.

[23] S. Baron and D. Kleinman. "The human as an optimal controller and information processor". In: Man-Machine Systems, IEEE Transactions on 10.1 (1969), pp. 917.

[24] G. Burnham, J. Seo, and G. Bekey. "Identification of human driver models in car following". In: Automatic Control, IEEE Transactions on 19.6 (1974), pp. 911-915.

[25] T. Mandal and Y. Gu. "Pilot-Vehicle System Modeling Using Sub-Scale Flight Experiments". In: ((2014)).

[26] A. Lunteren. "Identification of human operator describing function models with one or two inputs in closed loop systems". PhD thesis. TU Delft, Delft University of Technology, (1979).

[27] R. Merwe, E. Wan, S. Julier, et al. "Sigma-point Kalman filters for nonlinear estimation and sensor-fusion: Applications to integrated navigation". In: Proceedings of the AIAA Guidance, Navigation \& Control Conference. (2004), pp. 16-19.

[28] P. Zaal and B. Sweet. "Estimation of time-varying pilot model parameters". In: AIAA Modeling and Simulation Technologies Conference, Portland, OR (8-11 August 2011). (2011).

[29] P. Thompson, D. Klyde, and M. Brenner. "Wavelet-based time-varying human operator models". In: AIAA Paper 4009 (2001), pp. 6-9.

[30] James R Schiess and Vincent R Roland. "Kalman filter estimation of human pilotmodel parameters". In: NASA Technical Report, (1975).

[31] E. Wan, R. Merwe, et al. "The unscented Kalman filter for nonlinear estimation". In: Adaptive Systems for Signal Processing, Communications, and Control Symposium 2000. AS-SPCC. The IEEE 2000. IEEE. (2000), pp. 153-158.

[32] D. McRuer and E. Krendel. Mathematical models of human pilot behavior. Tech. rep. DTIC Document, (1974). 
[33] S. Julier and J. Uhlmann. "New extension of the Kalman filter to nonlinear systems'. In: AeroSense'97. International Society for Optics and Photonics. (1997), pp. $182-193$.

[34] M. Rhudy, Y. Gu, and M. Napolitano. "An analytical approach for comparing linearization methods in EKF and UKF". In: International Journal of Advanced Robotic Systems 10.208 (2013).

[35] R. Nelson. Flight stability and control. (1998).

[36] D. Mitchell, A. Arencibia, and S. Munoz. "Real-time detection of pilot-induced oscillations". In: AIAA Atmospheric Flight Mechanics Conference and Exhibit. (2004), p. 4700 .

[37] G. Chowdhary, S. Srinivasan, and E. Johnson. "Frequency domain method for realtime detection of oscillations". In: Journal of Aerospace Computing, Information, and Communication 8.2 (2011), pp. 42-52.

[38] D. Johnson. Suppression of pilot-induced oscillation (PIO). Tech. rep. DTIC Document, (2002).

[39] M. Pavel et al. "Adverse rotorcraft pilot couplings - past, present and future challenges". In: Progress in Aerospace Sciences 62 (2013), pp. 1-51.

[40] J. Slotine, W. Li, et al. Applied nonlinear control. Vol. 199. 1. prentice-Hall Englewood Cliffs, NJ, (1991).

[41] C. Fielding and PK. Flux. "Non-linearities in flight control systems". In: Aeronautical journal 107.1077 (2003), pp. 673-686.

[42] D. Klyde, D. McRuer, and T. Myers. "PIO analysis with actuator rate limiting". In: AIAA Atmospheric Flight Mechanics Conference. (1996), pp. 569-580. 